\title{
Bianchi attractors: a classification of extremal black brane geometries
}

\author{
Norihiro lizuka, ${ }^{a}$ Shamit Kachru, ${ }^{b}$ Nilay Kundu, ${ }^{c}$ Prithvi Narayan, ${ }^{c}$ Nilanjan Sircar ${ }^{c}$ \\ and Sandip P. Trivedi ${ }^{c}$
}

${ }^{a}$ Theory Division, CERN, CH-1211 Geneva 23, Switzerland

${ }^{b}$ Stanford Institute for Theoretical Physics, Department of Physics and Theory Group, SLAC National Accelerator Laboratory Stanford University, Palo Alto, CA 94305, U.S.A.

${ }^{c}$ Tata Institute for Fundamental Research, Homi Bhabha Road, Mumbai 400005, India

E-mail: norihiro.iizuka@cern.ch, skachru@stanford.edu, nilay.tifr@gmail.com, prithvi.narayan@gmail.com, nilanjan@theory.tifr.res.in, trivedi.sp@gmail.com

ABSTRACT: Extremal black branes are of interest because they correspond to the ground states of field theories at finite charge density in gauge/gravity duality. The geometry of such a brane need not be translationally invariant in the spatial directions along which it extends. A less restrictive requirement is that of homogeneity, which still allows points along the spatial directions to be related to each other by symmetries. In this paper, we find large new classes of homogeneous but anisotropic extremal black brane horizons, which could naturally arise in gauge/gravity dual pairs. In $4+1$ dimensional spacetime, we show that such homogeneous black brane solutions are classified by the Bianchi classification, which is well known in the study of cosmology, and fall into nine classes. In a system of Einstein gravity with negative cosmological term coupled to one or two massive Abelian gauge fields, we find solutions with an additional scaling symmetry, which could correspond to the near-horizon geometries of such extremal black branes. These solutions realize many of the Bianchi classes. In one case, we construct the complete extremal solution which asymptotes to AdS space.

KEYwords: Gauge-gravity correspondence, AdS-CFT Correspondence, Holography and condensed matter physics (AdS/CMT)

ARXIV EPRINT: 1201.4861 


\section{Contents}

1 Introduction 2

2 Generalised translations and the Bianchi classification $\quad 4$

2.1 The Bianchi classification 6

3 General set-up and a simple example $\quad 6$

3.1 A simple example based on Type VII 8

$\begin{array}{lll}3.1 .1 & \text { Some concluding remarks } & 10\end{array}$

4 Solutions of other Bianchi types $\quad 11$

$\begin{array}{lll}4.1 \text { Type II } & 12\end{array}$

4.2 Type VI, III and V 13

$\begin{array}{lll}\text { 4.2.1 Type } \mathrm{V} & 14\end{array}$

$\begin{array}{lll}4.2 .2 & \text { Type III } & 15\end{array}$

$\begin{array}{lll}4.3 & \text { Type IX and Type VIII } & 15\end{array}$

5 An extremal brane interpolating from Type VII to $A d S_{5} \quad 16$

$\begin{array}{ll}5.1 \text { The system and the essential idea } & 17\end{array}$

$\begin{array}{ll}5.2 \text { Type VII solutions } & 18\end{array}$

5.2.1 The limit 20

5.3 Extremal RN brane 21

5.4 The perturbation in Type VII 22

$\begin{array}{ll}5.5 & \text { The interpolation } \\ \end{array}$

6 Generalised translations involving time and closed time-like curves $\quad 26$

$\begin{array}{llr}7 & \text { Discussion } & 29\end{array}$

$\begin{array}{ll}\text { A Three dimensional homogeneous spaces } & 31\end{array}$

B Gauge field equation of motion $\quad 33$

B.1 Gauge field with components along spatial directions 34

B.2 Gauge field with components only along time 35

$\begin{array}{ll}\text { C Lifshitz solutions } & 36\end{array}$

$\begin{array}{ll}\text { D Extremal RN solution } & 37\end{array}$ 


\section{Introduction}

Nature can exist in many varied and beautiful phases. String theory too has many varied and beautiful phases, which correspond to the huge landscape of vacua in the theory. It is natural to hope that some of the phases of string theory might help describe those in Nature. This hope has spurred recent progress attempting, for example, to build connections between string theory and condensed matter physics.

Some of the work in this direction has involved the study of black branes in gravity theories, with a particular focus on their role as holographic duals to field theories at finite temperature and/or chemical potential. Extremal branes are particularly interesting, since they correspond to zero temperature ground states. Without a temperature quantum fluctuations come into "their own", especially in strongly correlated systems, leading to interesting and novel phenomena like quantum phase transitions. The description of these ground states in terms of an extremal brane allows such effects to be studied in a non-trivial and often tractable mean field approximation.

So far only a few different kinds of extremal brane solutions have been found. The analogy with the phases of matter, mentioned above, would suggest that many more should exist. The main point of this paper is to show that this expectation is indeed true. We will argue below that the known types of extremal black branes are only the "tip of the iceberg", and that there is a much bigger zoo of solutions, obtainable in theories of gravity with reasonable matter, waiting to be discovered.

Symmetries are a good place to start in classifying the solutions of general relativity and also in classifying the phases of matter. The known extremal solutions mostly have the usual translational symmetries along the spatial directions in which the brane extends. The solutions we consider here differ by having a generalised version of translational invariance. Any two points along the spatial directions can still be connected by a symmetry transformation, but the generators of the symmetries now do not necessarily commute with each other. This generalised notion of translations is well known in general relativity. Space-times with such symmetries are said to be homogeneous.

To be more precise, we will study brane solutions of the form

$$
d s^{2}=d r^{2}-g_{00} d t^{2}+g_{i j} d x^{i} d x^{j}
$$

where $i, j=1, \cdots d-1$ are the spatial directions along which the brane extends. We will find that often, for reasonable matter Lagrangians, there are brane solutions where the $x^{i}$ coordinates span a homogeneous space with isometries which do not commute. Most of our discussion will be for the case $d=4$ where the brane extends in 3 space dimensions. ${ }^{1}$ The different kinds of generalised translational symmetries that can arise along three spatial directions are well known in general relativity. They lie in the Bianchi classification and fall into 9 different classes, e.g., see [1,2]. These classes therefore classify all homogeneous brane solutions of the type eq. (1.1).

\footnotetext{
${ }^{1}$ Towards the end of the paper we will briefly discuss examples where the time direction could also be involved in the generalised translations.
} 
Of particular interest in the study of extremal branes is their near-horizon geometry. The near-horizon geometry encodes information about the low energy dynamics of the dual field theory and often turns out to be scale invariant. The near horizon geometry is also often an attractor, with differences from the attractor geometry far away dying out as one approaches the horizon. This feature corresponds to the fact that much of the UV data in the field theory is often irrelevant in the IR. These properties mean that the near-horizon geometry is often easier to find analytically than the full solution. The attractor nature also makes the near-horizon geometry more universal than the full extremal solution. Thus one is in the happy situation that the IR region, which being more universal is of greater interest anyway, is also the region one can obtain with relative ease.

In this paper we will focus mostly on the near-horizon region. We will consider solutions which are scale invariant in this region, along with being homogeneous along the spatial directions. ${ }^{2}$ We will show that solutions lying in many of the 9 Bianchi classes mentioned above can be obtained by coupling gravity to relatively simple kinds of matter. In the examples we consider, one or two massive Abelian gauge fields in the presence of a negative cosmological constant will suffice. In one case we will also find interpolating solutions for the full extremal brane which interpolate between the scaling near-horizon region and asymptotic AdS space.

A simple example of the kind of generalised translation invariance we have in mind arises in condensed matter physics when a non-zero momentum mode condenses, leading to a vector order parameter which varies in a helical manner in space with a pitch determined by the momentum of the condensed mode. For example, in spin systems this is known to happen when a spin wave of non-zero momentum condenses, resulting in the magnetisation order parameter varying in a helical pattern (see [3, 4] for a discussion). In superconductors, it has been argued that such spatially modulated phases can arise due to the FFLO instability [5, 6]. Similar behavior can also occur in QCD [7] and other systems [8]. It has also been discussed recently in the context of AdS/CFT in [9-12] and [13-15]. In the context of our discussion such situations lie in the Type VII class of the Bianchi classification. We will discuss this case below quite extensively since it is relatively simple and illustrates many of the features which arise in the other classes as well.

The paper is structured as follows. We begin with a discussion of the generalised translations and various Bianchi classes in section 2. Then we discuss one illustrative example of a Bianchi Type VII near-horizon geometry in section 3. Other examples giving rise to Bianchi Types II, III, V, VI, and IX are discussed in section 4. A concrete example where a Type VII near-horizon geometry can arise from an asymptotically AdS spacetime is given in section 5. A brief discussion of subtleties which might arise when time is involved in the generalised translations is contained in section 6 . The paper ends with some discussion in section 7. Important supplementary material is contained in the appendices A-D.

Before closing the introduction, let us comment on related literature. There is a formidable body of work on brane solutions in the string theory and general relativity

\footnotetext{
${ }^{2}$ The scaling symmetry will correspond to translations in the radial coordinate $r$. Thus, including the scaling symmetry makes the full $d+1$ dimensional space-time geometry homogeneous.
} 
literature; for a recent review with further references, see [16]. A classification of extremal black holes (as opposed to black branes), quite different from ours, has been discussed in [17]. Our solutions can be viewed as black branes with new kinds of "hair"; simple discussions of how branes in AdS space can violate the black hole no-hair theorems are given in the papers on holographic superconductivity, see e.g. [18] and [19] for discussions with additional references. Early examples of black branes with interesting horizon structure were discussed in studies of the Gregory-Laflamme instability; the original papers are [20, 21] and a recent discussion appears in [22]. As we mentioned previously, solutions with instabilities of the Type VII kind have appeared already in the context of AdS/CFT duality in the interesting papers [9-15]. Lifshitz symmetry has characterized one new type of horizon to emerge in holographic duals of field theories at finite charge density in many recent studies; this was discussed in [23] and [24] (see also [25-31] for string theory and supergravity embeddings of such solutions). The attractor mechanism has also inspired a considerable literature. The seminal paper is [32]. For a recent review with a good collection of references, see [33]. Some references on attractors without supersymmetry are [34-38]. A recent discussion of how the attractor mechanism may be related to new kinds of horizons for black branes, including e.g. Lifshitz solutions, appears in [39].

\section{Generalised translations and the Bianchi classification}

It is worth beginning with a simple example illustrating the kind of generalised translational symmetry we would like to explore. Suppose we are in three dimensional space described by coordinates $x^{1}, x^{2}, x^{3}$ and suppose the system of interest has the usual translational symmetries along the $x^{2}, x^{3}$ directions. It also has an additional symmetry but instead of being the usual translation in the $x^{1}$ direction it is now a translation accompanied by a rotation in the $x^{2}-x^{3}$ plane. The translations along the $x^{2}, x^{3}$ direction are generated by the vectors fields,

$$
\xi_{1}=\partial_{2}, \quad \xi_{2}=\partial_{3},
$$

whereas the third symmetry is generated by the vector field,

$$
\xi_{3}=\partial_{1}+x^{2} \partial_{3}-x^{3} \partial_{2}
$$

It is easy to see that the three transformations generated by these vectors transform any point in the three dimensional plane to any other point in its immediate neighborhood. This property, which is akin to that of usual translations, is called homogeneity. However, the symmetry group we are dealing with here is clearly different from usual translations, since the commutators of the generators, eq. (2.1), (2.2) take the form

$$
\left[\xi_{1}, \xi_{2}\right]=0 ; \quad\left[\xi_{1}, \xi_{3}\right]=\xi_{2} ; \quad\left[\xi_{2}, \xi_{3}\right]=-\xi_{1},
$$

and do not vanish, as they would have for the usual translations. ${ }^{3}$

\footnotetext{
${ }^{3}$ In fact this symmetry group is simply the group of symmetries of the two dimensional Euclidean plane, its two translations and one rotation.
} 
In this paper we will be interested in exploring such situations in more generality where the symmetries are different from the usual translations but still preserve homogeneity, allowing any point in the system of interest to be transformed to any other by a symmetry transformation. ${ }^{4}$

When can one expect such a symmetry group to arise? Let us suppose that there is a scalar order parameter, $\phi(x)$, which specifies the system of interest. Then this order parameter must be invariant under the unbroken symmetries. The change of this order parameter under an infinitesimal transformation generated by the vector field $\xi_{i}$ is given by

$$
\delta \phi=\epsilon \xi_{i}(\phi)
$$

and this would have to vanish for the transformation to be a symmetry. It is easy to see that requiring that this is true for the three symmetry generators mentioned above, eq. (2.1), eq. (2.2), leads to the condition that $\phi$ is a constant independent of the coordinates, $x^{1}, x^{2}, x^{3}$. Now, such a constant configuration for $\phi$ is invariant under the usual translation along $x^{1}$, generated by $\partial_{1}$, besides being invariant also under a rotation in the $x^{2}-x^{3}$ plane (and the other rotations). Thus we see that with a scalar order parameter a situation where the generalised translations are unbroken might as well be thought of as one that preserves the usual three translations along with additional rotations.

Now suppose that instead of being a scalar the order parameter specifying the system is a vector $V$, which we denote by $V^{i} \partial_{i}$. Under the transformation generated by a vector field $\xi_{i}$ this transforms as

$$
\delta V=\epsilon\left[\xi_{i}, V\right]
$$

Requiring that these commutators vanish for all three $\xi_{i}$ 's leads to the conditions,

$$
V^{1}=\text { constant }, \quad V^{2}=V_{0} \cos \left(x^{1}+\delta\right), V^{3}=V_{0} \sin \left(x^{1}+\delta\right)
$$

where $V_{0}, \delta$ are constants independent of all coordinates. In other words the $V^{2}, V^{3}$ components are not constant but rotate in the $2-3$ plane as one advances along the $x^{1}$ direction. In contrast, for the usual translations all three components $V^{1}, V^{2}, V^{3}$ would be constant and the behavior of the order parameter would be different. Thus we see that with a vector order parameter, instead of a scalar, one can have situations where the generalised translational symmetries generated by eq. (2.1), eq. (2.2) are preserved but the usual translation group is not. Such a situation could also arise if the order parameter were a tensor field.

In fact situations with this type of a vector order parameter are well known to arise in condensed matter physics as was mentioned in the introduction. For example this can happen in spin systems, where the order parameter is the magnetisation. In the presence of parity violation in such a system a helical spin density wave of appropriate wavelength can be set up $[3,4]$.

\footnotetext{
${ }^{4}$ In a connected space this follows from the requirement that any point is transformed to any other point in its immediate neighborhood by a symmetry transformation.
} 


\subsection{The Bianchi classification}

The example discussed above shares many features in common with other symmetry groups which preserve homogeneity. Luckily all such groups in three dimensions have been classified and are easily found in the physics literature since they have been of interest in the study of homogeneous situations in cosmology $[1,2]$. It is known that there are 9 such inequivalent groups and they are described by the Bianchi classification. The idea behind this classification is quite simple. As we have seen above the Killing vectors which generate the symmetries form a Lie Algebra. Homogeneity requires that there should be 3 such Killing vectors in 3 dimensions. The 9 groups, and related 9 classes in the Bianchi classification, simply correspond to the 9 inequivalent real Lie Algebras one can get with 3 generators.

The familiar case of translational invariance, which includes AdS space and Lifshitz spacetimes, corresponds to the case where the three generators commute and is called Bianchi Class I. The remaining 8 classes are different from this one and give rise to new kinds of generalised translational symmetries. The symmetry group discussed above in eq. (2.1), eq. (2.2) falls within Class VII in the Bianchi classification, with the parameter $h=0$ in the notation of [2], see page 112. We will refer to this case as Type $V I I_{0}$ below. As mentioned above it corresponds to the group of symmetries of the two-dimensional Euclidean plane. Like the example above, in the more general case as well, a scalar order parameter will not lead to a situation where the usual translations are broken. Instead a vector or more generally a tensor order parameter is needed. Such an order parameter, when it takes a suitable form, can preserve the symmetry group characterizing any of the other 8 classes.

In appendix A we give some details of the algebras for all 9 Bianchi classes. In our discussion below we will consider specific examples which lie in the Bianchi Classes, II, III, V, VI, VII and IX. Some extra details on these classes, including the generators, invariant one-forms etc, can also be found in appendix A.

\section{General set-up and a simple example}

Here we turn to describing more explicitly a spacetime metric incorporating the generalised translational symmetries described above and to asking when such a metric can arise as the solution to Einstein's equations of gravity coupled with matter. Mostly we will work in 5 dimensions, this corresponds to setting $d=4$ in eq. (1.1). In addition, to keep the discussion simple, we assume that the usual translational symmetry along the time direction is preserved so that the metric is time independent and also assume that there are no off-diagonal components between $t$ and the other directions in the metric. This leads to

$$
d s^{2}=d r^{2}-a(r)^{2} d t^{2}+g_{i j} d x^{i} d x^{j}
$$

with the indices $i, j$ taking values $1,2,3$. For any fixed value of $r, t$, we get a three dimensional subspace spanned by $x^{i}$. We will take this subspace to be a homogeneous space, corresponding to one of the 9 types in the Bianchi classification. 
As discussed in [2], for each of the 9 cases there are three invariant one forms, $\omega^{i}, i=$ $1,2,3$, which are invariant under all 3 isometries. A metric expressed in terms of these oneforms with $x^{i}$ independent coefficients will be automatically invariant under the isometries. For future reference we also note that these one-forms satisfy the relations

$$
d \omega^{i}=\frac{1}{2} C_{j k}^{i} \omega^{j} \wedge \omega^{k}
$$

where $C_{j k}^{i}$ are the structure constants of the group of isometries [2].

We take the metric to have one additional isometry which corresponds to scale invariance. An infinitesimal isometry of this type will shift the radial coordinate by $r \rightarrow r+\epsilon$. In addition we will take it to rescale the time direction with weight $\beta_{t}, t \rightarrow t e^{-\beta_{t} \epsilon}$, and assume that it acts on the spatial coordinates $x^{i}$ such that the invariant one forms, $\omega^{i}$ transform with weights $\beta_{i}$ under it, ${ }^{5} \omega^{i} \rightarrow e^{-\beta_{i} \epsilon} \omega^{i}$.

These properties fix the metric to be of the form

$$
d s^{2}=R^{2}\left[d r^{2}-e^{2 \beta_{t} r} d t^{2}+\eta_{i j} e^{\left(\beta_{i}+\beta_{j}\right) r} \omega^{i} \otimes \omega^{j}\right]
$$

with $\eta_{i j}$ being a constant matrix which is independent of all coordinates.

In the previous section we saw that a situation where the usual translations were not preserved but the generalised translations were unbroken requires a vector (or possibly tensor) order parameter. A simple setting in gravity, which could lead to such a situation, is to consider an Abelian gauge field coupled to gravity. We will allow the gauge field to have a mass in the discussion below. Such a theory with a massive Abelian gauge field is already known to give rise to Lifshitz spacetimes [23, 24]. Here we will find that it has in fact an even richer set of solutions, exhibiting many possible phases and patterns of symmetries, as parameters are varied.

The action of the system we consider is therefore

$$
S=\int d^{5} x \sqrt{-g}\left\{R+\Lambda-\frac{1}{4} F^{2}-\frac{1}{4} m^{2} A^{2}\right\}
$$

and has two parameters, $m^{2}, \Lambda$. We note that in our conventions $\Lambda>0$ corresponds to AdS space.

The three isometries along the $x^{i}$ directions, time translation and scale invariance restrict the form for the gauge potential to be ${ }^{6}$

$$
A=A_{t} e^{\beta_{t} r} d t+A_{i} e^{\beta_{i} r} \omega^{i}
$$

where $A_{t}, A_{i}$ are constants independent of all coordinates.

\footnotetext{
${ }^{5}$ The dilatation generator could act more generally than this. A discussion of the more general case is left for the future.

${ }^{6}$ A possible $A_{r} d r$ term, where $A_{r}$ only depends on $r$ to be consistent with the symmetries, can always be gauged away.
} 


\subsection{A simple example based on Type VII}

To construct a simple and explicit example we now return to the case of Type $V I I_{0}$ with which we began our discussion of generalised translational symmetry in section 2 . We will show here that a metric with this type of symmetry can arise as a solution to Einstein's equations.

The one-forms which are invariant under the isometries for this case are given, see [2], page 112 , by

$$
\omega^{1}=d x^{1} ; \omega^{2}=\cos \left(x^{1}\right) d x^{2}+\sin \left(x^{1}\right) d x^{3} ; \omega^{3}=-\sin \left(x^{1}\right) d x^{2}+\cos \left(x^{1}\right) d x^{3} .
$$

As discussed above we are assuming that the $x^{i}$ coordinates transform under the scaling isometry in such a way that the one-forms, $\omega^{i}$, transform with definite weights. It is clear from eq. (3.6) that the only way this can happen is if $x^{1}$ is invariant, and $\left(x^{2}, x^{3}\right) \rightarrow$ $e^{-\beta \epsilon}\left(x^{2}, x^{3}\right)$, under $r \rightarrow r+\epsilon$, so that $\omega^{2}, \omega^{3}$ have equal weights and transform as $\left(\omega^{2}, \omega^{3}\right) \rightarrow$ $e^{-\beta \epsilon}\left(\omega^{2}, \omega^{3}\right)$. To keep the discussion simple we also assume that the metric coefficients $\eta_{i j}$ which appear in eq. (3.3) are diagonal. After some rescaling this allows the metric to be written as

$$
d s^{2}=R^{2}\left[d r^{2}-e^{2 \beta_{t} r} d t^{2}+\left(d x^{1}\right)^{2}+e^{2 \beta r}\left(\left(\omega^{2}\right)^{2}+\lambda^{2}\left(\omega^{3}\right)^{2}\right)\right] .
$$

The only unknowns that remain in the metric are the two constants, $R, \lambda$ and the two scaling weights, $\beta_{t}$ and $\beta$.

Before proceeding let us comment on the physical significance of the parameter $R$. From eq. (3.6) we see that the coordinate distance in the $x^{1}$ direction needed to complete one full rotation in the $x^{2}-x^{3}$ plane is $2 \pi$. From eq. (3.7) it follows that the physical distance along the $x^{1}$ direction that is needed is $2 \pi R$. Thus $R$ determines the pitch of the helix.

We will see below that a metric of the type in eq. (3.7) arises as a solution for the system, eq. (3.4), when the gauge field takes the form

$$
A=e^{\beta r}\left(\sqrt{\tilde{A}_{2}} \omega^{2}+\sqrt{\tilde{A}_{3}} \omega^{3}\right) .
$$

Let us begin with the gauge field equation of motion

$$
d *_{5} F=-\frac{1}{2} m^{2} *_{5} A .
$$

As discussed in appendix B this gives rise to the conditions eq. (B.19), eq. (B.20) (with the index $i$ taking values 2,3). For the metric eq. (3.7), eq. (B.19) is met. From eq. (B.13), (B.17), (B.18) in appendix B, and also from [2], page 112, we have $k^{1}=0, k^{2}=$ $k^{3}=-1$. Also comparing eq. (3.7) and eq. (B.3) we see that $\lambda_{1}=\lambda_{2}=1, \lambda_{3}=\lambda$, and $\beta_{1}=0, \beta_{2}=\beta_{3}=\beta$. If both $\tilde{A}_{2}$ and $\tilde{A}_{3}$ are non-vanishing eq. (B.20) then gives

$$
\begin{aligned}
& \lambda^{2}\left(m^{2}-2 \beta\left(\beta+\beta_{t}\right)\right)+2=0 \\
& \left(m^{2}-2 \beta\left(\beta+\beta_{t}\right)\right)+2 \lambda^{2}=0 .
\end{aligned}
$$


Assuming $\lambda \neq 1$ these conditions cannot both be met. Thus we are lead to conclude that either $\tilde{A}_{2}$ or $\tilde{A}_{3}$ must vanish. We will set $\tilde{A}_{3}=0$ without loss of generality, ${ }^{7}$ so that the gauge field is given by

$$
A=\sqrt{\tilde{A}_{2}} e^{\beta r} \omega^{2}
$$

and the gauge field EOM gives

$$
\lambda^{2}\left(m^{2}-2 \beta\left(\beta+\beta_{t}\right)\right)+2=0 .
$$

Note that the solution we are seeking has five parameters, $R, \beta_{t}, \beta, \lambda$, which enter in the metric and $\tilde{A}_{2}$, which determines the gauge field. These are all constants.

Next, turn to the Einstein equations

$$
R_{\nu}^{\mu}-\frac{1}{2} \delta_{\nu}^{\mu} R=T_{\nu}^{\mu}
$$

with

$$
T_{\nu}^{\mu}=\frac{1}{2} F_{\lambda}^{\mu} F_{\nu}^{\lambda}+\frac{1}{4} m^{2} A^{\mu} A_{\nu}+\frac{1}{2} \delta_{\nu}^{\mu}\left(\Lambda-\frac{1}{4} F^{\rho \sigma} F_{\rho \sigma}-\frac{1}{4} m^{2} A^{\rho} A_{\rho}\right)
$$

Along the $t$, $r r$, and $x^{1} x^{1}$ directions these give:

$$
\begin{array}{r}
\frac{2\left(1+\tilde{A}_{2}\right)}{\lambda^{2}}+2 \lambda^{2}+\tilde{A}_{2}\left(m^{2}+2 \beta^{2}\right)+24 \beta^{2}-4(1+\Lambda)=0 \\
\frac{2\left(1+\tilde{A}_{2}\right)}{\lambda^{2}}+2 \lambda^{2}+\tilde{A}_{2}\left(m^{2}-2 \beta^{2}\right)+8 \beta\left(\beta+2 \beta_{t}\right)-4(1+\Lambda)=0 \\
\frac{2\left(1+\tilde{A}_{2}\right)}{\lambda^{2}}+2 \lambda^{2}-\tilde{A}_{2}\left(m^{2}+2 \beta^{2}\right)-8\left(3 \beta^{2}+2 \beta_{t} \beta+\beta_{t}^{2}\right)-4(1-\Lambda)=0 .
\end{array}
$$

The components along the $x^{2}, x^{3}$ directions lead to

$$
\begin{aligned}
& \frac{2\left(3+\tilde{A}_{2}\right)}{\lambda^{2}}-2 \lambda^{2}-\tilde{A}_{2}\left(m^{2}+2 \beta^{2}\right)+8\left(\beta^{2}+\beta_{t} \beta+\beta_{t}^{2}\right)-4(1+\Lambda)=0 \\
& \frac{2\left(1+\tilde{A}_{2}\right)}{\lambda^{2}}-6 \lambda^{2}-\tilde{A}_{2}\left(m^{2}+2 \beta^{2}\right)-8\left(\beta^{2}+\beta_{t} \beta+\beta_{t}^{2}\right)+4(1+\Lambda)=0 .
\end{aligned}
$$

In obtaining these equations we have set $R$, which appears as the overall scale in front of the metric eq. (3.7), to be unity. The equations can then be thought of as determining the dimensionful parameters $\Lambda, m^{2}, \tilde{A}_{2}$ in terms of $R$. Counting eq. (3.13) these are 6 equations in all. One can check that only 5 of these are independent. These 5 equations determine the 5 parameters $\left(\Lambda, \beta, \beta_{t}, \lambda, \tilde{A}_{2}\right)$, which then completely determines the solution.

\footnotetext{
${ }^{7}$ From eq. (3.7) and eq. (3.6) we see that when $\lambda=1$ the symmetries in the $\left(x^{1}, x^{2}, x^{3}\right)$ directions are enhanced to the usual translations and rotations.
} 
Solving, we get:

$$
\begin{aligned}
m^{2} & =\frac{2\left(11+2 \lambda^{2}-10 \lambda^{4}+3 \lambda^{6}\right)}{\lambda^{2}\left(5 \lambda^{2}-11\right)} \\
\Lambda & =\frac{1}{50}\left(95 \lambda^{2}+\frac{25}{\lambda^{2}}-\frac{50}{\lambda^{2}-2}+\frac{144}{5 \lambda^{2}-11}-146\right) \\
\beta & =\sqrt{2} \sqrt{\frac{2-3 \lambda^{2}+\lambda^{4}}{5 \lambda^{2}-11}} \\
\beta_{t} & =\frac{\lambda^{2}-3}{\sqrt{2}\left(\lambda^{2}-2\right)} \sqrt{\frac{2-3 \lambda^{2}+\lambda^{4}}{5 \lambda^{2}-11}} \\
\tilde{A}_{2} & =\frac{2}{2-\lambda^{2}}-2 .
\end{aligned}
$$

The first of these equations can be thought of as determining $\lambda$ in terms of $m^{2}$, the second then as determining $\Lambda$ in terms of $m^{2}$, and so on, all in units where $R=1$.

Requiring $\tilde{A}_{2}>0$ gives

$$
1 \leq \lambda<\sqrt{2}
$$

Actually eq. (3.23) and eq. (3.24) only determine $\beta$ and $\beta_{t}$ upto an overall sign. We have chosen the sign so that in the range eq. (3.26) $\beta_{t}$ are positive, and the $g_{t t}$ component of the metric in particular vanishes at the horizon, $r \rightarrow-\infty$. Also we note that in this range, $m^{2}$ is a monotonic function of $\lambda$, and takes values in the range $-2 \leq m^{2}<1$, where $m^{2}(\lambda=1)=-2$.

\subsubsection{Some concluding remarks}

We end this section with some concluding remarks.

An important question which remains is about the stability of the solution we have found above. We leave this for future study except to note that in general, negative values of $m^{2}$ do not necessarily imply an instability, as is well known from the study of AdS space, as long as the magnitude of $m^{2}$ is not very large.

It is also worth comparing our solution to the well known Lifshitz solution obtained from the same starting point eq. (3.4). This solution is discussed in appendix C for completeness. We see that with a spatially oriented gauge field the Lifshitz solution can arise only if $m^{2}$ is positive, eq. (C.15). In the parametric range $1>m^{2}>0$ both the Type $V I I_{0}$ solution and the Lifshitz solution are allowed.

Finally, it is worth noting that like the Lifshitz case, [41], while curvature invariants are finite in the solution found above the tidal forces experienced by a freely falling observer can diverge at the horizon. The curvature invariants for the solution take values

$$
\begin{aligned}
& R_{\mu}^{\mu}=\frac{123}{25}+\frac{72}{275-125 \lambda^{2}}+\frac{1}{\lambda^{2}-2}-\frac{5+39 \lambda^{4}}{10 \lambda^{2}} \\
& R^{\mu \nu} R_{\mu \nu}=\frac{\left(\lambda^{2}-1\right)^{2}}{4 \lambda^{4}\left(22-21 \lambda^{2}+5 \lambda^{4}\right)^{2}}\left(1452-1804 \lambda^{2}+8229 \lambda^{4}\right. \\
&\left.-14304 \lambda^{6}+10114 \lambda^{8}-3204 \lambda^{10}+381 \lambda^{12}\right)
\end{aligned}
$$




$$
\begin{array}{r}
R^{\mu \nu \sigma \rho} R_{\mu \nu \sigma \rho}=\frac{\left(\lambda^{2}-1\right)^{2}}{4 \lambda^{4}\left(22-21 \lambda^{2}+5 \lambda^{4}\right)^{2}}\left(5324-6732 \lambda^{2}+12063 \lambda^{4}\right. \\
\left.-19128 \lambda^{6}+14126 \lambda^{8}-4700 \lambda^{10}+583 \lambda^{12}\right)
\end{array}
$$

and are indeed finite. ${ }^{8}$ To see that the tidal forces can diverge we note that a family of geodesics can be found for the metric eq. (3.7) where $x^{1}, x^{2}, x^{3}$ take any constant values and $(r, t)$ vary with a functional dependence on the proper time, $\tau$, given by $(r(\tau), t(\tau))$ that is independent of these constant values. Now take any two geodesics with the same $(r(\tau), t(\tau))$ which are separated along $x^{2}, x^{3}$. The proper distance separating them in the $x^{2}, x^{3}$ directions vanishes rapidly as the geodesics go to the horizon, $r \rightarrow-\infty$ leading to a diverging tidal force.

It is worth trying to find the small temperature deformation of the Type VII solution above. Such a deformation will probably allow us to control the effects of these diverging tidal forces.

\section{Solutions of other Bianchi types}

The discussion so far has mostly focused on examples of Bianchi Type VII. Solutions which lie in Bianchi Type I, e.g. Lifshitz solutions, are of course well known [23, 24]. In this section we will give examples of some of the other Bianchi types. Our purpose in this section is not to give a very thorough or complete discussion of these other classes, but rather to illustrate how easy it is to obtain solutions of these other types as well.

As in the discussion in section 3 for Type VII we focus on solutions which have in addition the usual time translational invariance as well as the scaling symmetry involving a translation in the radial direction. The presence of the scaling symmetry will simplify the analysis. Also, such scaling solutions are often the attractor end points of more complete extremal black brane geometries. Accordingly we expect the scaling solutions we discuss to be more general than the specific systems we consider, and to arise in a wide variety of settings.

A remarkable feature is that so many different types of solutions can be obtained from relatively simple gravity + matter Lagrangians. Mostly, we will consider the system described in eq. (3.4) consisting of only one massive Abelian gauge field and gravity. Towards the end, to get a non-trivial Type IX case, we will consider two massive gauge fields.

Our analysis has two important limitations. We will not investigate the stability of these solutions. And we will not investigate whether the scaling solutions discussed in this section can be obtained as IR end points of more general geometries which are say asymptotically AdS. We leave these questions for the future. For the Type VII case discussed in the previous section, we will construct an example in section 5 of a full extremal solution which asymptotes to AdS space.

The discussion in this section is a bit brief since it shares many similarities with the Type VII case studied in section 3.

\footnotetext{
${ }^{8}$ All three of the curvature scalar diverges at $\lambda=0, \pm \sqrt{2}, \pm \sqrt{\frac{11}{5}}$, but these values lie outside the range of validity of the solution given by eq. (3.26).
} 


\subsection{Type II}

We begin by considering an example which lies in Type II. The system we consider is given by eq. (3.4). The symmetry group for Type II is the Heisenberg group. The generators in a convenient basis are given in appendix A. The invariant one-forms are

$$
\omega^{1}=d x^{2}-x^{1} d x^{3}, \quad \omega^{2}=d x^{3}, \quad \omega^{3}=d x^{1} .
$$

We take the metric to be of the form eq. (3.3) and diagonal,

$$
d s^{2}=R^{2}\left[d r^{2}-e^{2 \beta_{t} r} d t^{2}+e^{2\left(\beta_{2}+\beta_{3}\right) r}\left(\omega^{1}\right)^{2}+e^{2 \beta_{2} r}\left(\omega^{2}\right)^{2}+e^{2 \beta_{3} r}\left(\omega^{3}\right)^{2}\right] .
$$

We take the massive gauge field to be along the time direction

$$
A=\sqrt{A_{t}} e^{\beta_{t} r} d t
$$

As in the discussion of section 3.1 we will find it convenient to set $R=1$. The gauge field equation of motion is

$$
m^{2}-4\left(\beta_{2}+\beta_{3}\right) \beta_{t}=0 .
$$

The rr, tt, 11, 22, 33 components of the trace reversed Einstein equations ${ }^{9}$ are:

$$
\begin{aligned}
6\left(\beta_{2}^{2}+\beta_{2} \beta_{3}+\beta_{3}^{2}\right)-\left(A_{t}-3\right) \beta_{t}^{2}-\Lambda & =0 \\
A_{t}\left(3 m^{2}+4 \beta_{t}^{2}\right)+4\left(-3 \beta_{t}\left(2\left(\beta_{2}+\beta_{3}\right)+\beta_{t}\right)+\Lambda\right) & =0 \\
3+12 \beta_{3}\left(\beta_{2}+\beta_{3}\right)+6 \beta_{3} \beta_{t}+A_{t} \beta_{t}^{2}-2 \Lambda & =0 \\
12\left(\beta_{2}+\beta_{3}\right)^{2}+6\left(\beta_{2}+\beta_{3}\right) \beta_{t}+A_{t} \beta_{t}^{2}-3-2 \Lambda & =0 \\
3+12 \beta_{2}\left(\beta_{2}+\beta_{3}\right)+6 \beta_{2} \beta_{t}+A_{t} \beta_{t}^{2}-2 \Lambda & =0 .
\end{aligned}
$$

We have 6 parameters: $m^{2}, \Lambda, \beta_{t}, \beta_{2}, \beta_{3}$, and $A_{t}$. There are 5 independent equations, so we will express the 5 other parameters in terms of $\beta_{t}$.

The solution is given as,

$$
\begin{aligned}
m^{2}=\beta_{t}\left(-\beta_{t}+\sqrt{16+\beta_{t}^{2}}\right), & \Lambda=\frac{1}{16}\left(72+19 \beta_{t}^{2}-3 \beta_{t} \sqrt{16+\beta_{t}^{2}}\right), \\
A_{t}=\frac{19}{8}-\frac{3 \sqrt{16+\beta_{t}^{2}}}{8 \beta_{t}}, & \beta_{2}=\beta_{3}=\frac{1}{8}\left(-\beta_{t}+\sqrt{16+\beta_{t}^{2}}\right) .
\end{aligned}
$$

Note that $A_{t} \geq 0, \beta_{t}>0$ implies

$$
\beta_{t} \geq \frac{3}{\sqrt{22}}
$$

${ }^{9}$ Trace reversed Einstein equations in $d+1$ space-time dimensions are

$$
R_{\nu}^{\mu}=T_{\nu}^{\mu}-\frac{1}{d-1} \delta_{\nu}^{\mu} T, \quad T=T_{\mu \nu} g^{\mu \nu} .
$$




\subsection{Type VI, III and V}

Next we consider the three classes Type VI, III and V. Type VI is characterized by one parameter, $h$. The Killing vectors take the form, see appendix A,

$$
\xi_{1}=\partial_{2}, \xi_{2}=\partial_{3}, \xi_{3}=\partial_{1}+x^{2} \partial_{2}+h x^{3} \partial_{3} .
$$

When $h=0$ we get Type III and when $h=1$ we get Type V. Thus these two classes can be though of as limiting cases of Type VI.

Looking at the Killing vectors in eq. (4.13) we see that translations along the $x^{2}, x^{3}$ directions are of the conventional kind, but a translation along $x^{1}$ is accompanied by a rescaling along both $x^{2}$ and $x^{3}$ with weights unity and $h$ respectively.

The invariant one-forms are

$$
\omega^{1}=e^{-x^{1}} d x^{2}, \omega^{2}=e^{-h x^{1}} d x^{3}, \omega^{3}=d x^{1} .
$$

We consider again the system of a massive Abelian gauge field, eq. (3.4). We take the metric to be

$$
d s^{2}=R^{2}\left[d r^{2}-e^{2 \beta_{t} r} d t^{2}+\left(\omega^{3}\right)^{2}+e^{2 \beta_{1} r}\left(\omega^{1}\right)^{2}+e^{2 \beta_{2} r}\left(\omega^{2}\right)^{2}\right]
$$

and the gauge field to be

$$
A=\sqrt{A_{t}} e^{\beta_{t} r} d t
$$

We also set $R=1$ below.

The gauge field equation of motion is then

$$
m^{2}-2\left(\beta_{1}+\beta_{2}\right) \beta_{t}=0 .
$$

The rr, tt, 11, 22, 33 component of the trace reversed Einstein equations are

$$
\begin{aligned}
3 \beta_{1}^{2}+3 \beta_{2}^{2}-\left(A_{t}-3\right) \beta_{t}^{2}-\Lambda & =0 \\
\beta_{t}\left(\beta_{1}+\beta_{2}+\beta_{t}\right)-\frac{1}{12}\left(3 A_{t} m^{2}+4 A_{t} \beta_{t}^{2}+4 \Lambda\right) & =0 \\
6+6 h+6 \beta_{1}\left(\beta_{1}+\beta_{2}\right)+6 \beta_{1} \beta_{t}+A_{t} \beta_{t}^{2}-2 \Lambda & =0 \\
6 h+6 h^{2}+6 \beta_{2}\left(\beta_{1}+\beta_{2}\right)+6 \beta_{2} \beta_{t}+A_{t} \beta_{t}^{2}-2 \Lambda & =0 \\
6+6 h^{2}+A_{t} \beta_{t}^{2}-2 \Lambda & =0 .
\end{aligned}
$$

Whereas the r3 component of Einstein's equation gives

$$
\beta_{1}+h \beta_{2}=0 .
$$

The resulting solution is conveniently expressed as follows:

$$
\begin{aligned}
m^{2} & =2(1-h)^{2}\left(1-\beta_{2}^{2}\right) \\
\Lambda & =4 h+\frac{(1-h)^{2}}{\beta_{2}^{2}}+2\left(1-h+h^{2}\right) \beta_{2}^{2} \\
A_{t} & =\frac{2(1-h)^{2}-4\left(1-h+h^{2}\right) \beta_{2}^{2}}{(1-h)^{2}\left(1-\beta_{2}^{2}\right)}
\end{aligned}
$$




$$
\begin{aligned}
& \beta_{1}=-h \beta_{2} \\
& \beta_{t}=\frac{(1-h)\left(1-\beta_{2}^{2}\right)}{\beta_{2}} .
\end{aligned}
$$

Choosing the radial coordinate so that the horizon lies at $r \rightarrow-\infty$, we get $\beta_{t}>0$. If we require that $\beta_{1}, \beta_{2} \geq 0$ also, and impose that $A_{t} \geq 0$, as is required for the solution to exist, we get the constraints:

$$
h \leq 0 \quad \text { and } \quad 0<\beta_{2} \leq \frac{1-h}{\sqrt{2} \sqrt{1-h+h^{2}}} .
$$

Having chosen a value of $h$ which satisfies eq. (4.29) and thus a Bianchi Type VI symmetry, we can then pick a value of $\beta_{2}$ also meeting eq. (4.29). The rest of the equations can then be thought of as follows. Eq. (4.25) determines $R$ in units of $\Lambda$, eq. (4.24) then determines $m^{2}$ required to obtain this value of $\beta_{2}$ and the remaining equations determine the other parameters that enter in the solution.

Let us briefly comment on the limits which give Type V and Type III next.

\subsubsection{Type V}

To obtain this class we need to take the limit $h \rightarrow 1$. To obtain a well defined limit where $A_{t}$ does not blow up requires, eq. (4.26), that $\beta_{2} \rightarrow 0$ keeping $\beta_{t} /(1-h)$ fixed.

It is easy to see that the solution can then be expressed as follows: $m^{2} \rightarrow 0, \beta_{1}=0$ and

$$
\Lambda=4+\beta_{t}^{2} \quad \text { and } \quad A_{t}=2 \frac{\beta_{t}^{2}-2}{\beta_{t}^{2}}
$$

with the condition,

$$
\beta_{t}^{2} \geq 2
$$

After a change of coordinates $x^{1}=\log (\rho)$ the metric becomes,

$$
d s^{2}=\left[d r^{2}-e^{2 \beta_{t} r} d t^{2}\right]+\left[\frac{d \rho^{2}+\left(d x^{2}\right)^{2}+\left(d x^{3}\right)^{2}}{\rho^{2}}\right] .
$$

We see that this is simply $A d S_{2} \times E A d S_{3}$, where $E A d S_{3}$ denotes the three-dimensional space of constant negative curvature obtained from the Euclidean continuation of $A d S_{3}$.

The resulting limit is in fact a one parameter family of solutions determined by the charge density. To see this note that one can think of the first equation in eq. (4.30) as determining $R$ in terms of $\Lambda$, and the second equation as determining $\beta_{t}$ in terms of $A_{t}$ which fixes the charge density. $R$ determines the radius of $E A d S_{3}$ and $\beta_{t}$ then determines the radius of $A d S_{2}$.

Also, in this example the symmetries of the spatial manifold are enhanced from those of Type $\mathrm{V}$ to the full $\mathrm{SO}(3,1)$ symmetry group of $E A d S_{3} \cdot{ }^{10}$

\footnotetext{
${ }^{10}$ One natural way in which such a solution can be obtained is as the near horizon geometry of an extremal black brane in asymptotic $A d S_{5}$ space with non-normalizable metric deformations turned on so that the boundary theory lives in $E A d S_{3}$ space. One expects that the near- horizon geometry can arise without such a metric non-normalizable deformation being turned on as well.
} 


\subsubsection{Type III}

The limit $h \rightarrow 0$ is straightforward. One gets

$$
\begin{aligned}
m^{2} & =2\left(1-\beta_{2}^{2}\right) \\
\Lambda & =\frac{1}{\beta_{2}^{2}}+2 \beta_{2}^{2} \\
A_{t} & =\frac{2-4 \beta_{2}^{2}}{1-\beta_{2}^{2}} \\
\beta_{1} & =0 \\
\beta_{t} & =\frac{1-\beta_{2}^{2}}{\beta_{2}} .
\end{aligned}
$$

The metric is

$$
d s^{2}=d r^{2}-e^{2 \beta_{t} r} d t^{2}+e^{2 \beta_{2} r}\left(d x^{3}\right)^{2}+\frac{1}{\rho^{2}}\left(d \rho^{2}+\left(d x^{2}\right)^{2}\right)
$$

where $x^{1}=\log (\rho)$. We see that $\rho, x^{2}$ span two dimensional $E A d S_{2}$ space.

\subsection{Type IX and Type VIII}

In the Type IX case the symmetry group is $\mathrm{SO}(3)$. The invariant one-forms are,

$$
\begin{aligned}
\omega^{1} & =-\sin \left(x^{3}\right) d x^{1}+\sin \left(x^{1}\right) \cos \left(x^{3}\right) d x^{2} \\
\omega^{2} & =\cos \left(x^{3}\right) d x^{1}+\sin \left(x^{1}\right) \sin \left(x^{3}\right) d x^{2} \\
\omega^{3} & =\cos \left(x^{1}\right) d x^{2}+d x^{3} .
\end{aligned}
$$

We consider a metric ansatz

$$
d s^{2}=d r^{2}-e^{2 \beta_{t} r} d t^{2}+\left(\omega^{1}\right)^{2}+\left(\omega^{2}\right)^{2}+\lambda\left(\omega^{3}\right)^{2} .
$$

This corresponds to $A d S_{2} \times$ Squashed $\mathrm{S}^{3}$. We take the system to have two gauge fields. One will be massless with only its time component turned on, and the other will have mass $m^{2}$ and an expectation value proportional to the one-form $\omega^{3}$ :

$$
A_{1}=\sqrt{A_{t}} e^{\beta_{t} r} d t, \quad A_{2}=\sqrt{A_{s}} \omega^{3}=\sqrt{A_{s}}\left(\cos \left(x^{1}\right) d x^{2}+d x^{3}\right) .
$$

The equation of motion for the first gauge field is automatically satisfied, while the equation of motion for the second yields

$$
m^{2}+2 \lambda=0
$$

The independent trace reversed Einstein equations are

$$
\begin{aligned}
A_{s}+2\left[\left(A_{t}-3\right) \beta_{t}^{2}+\Lambda\right] & =0 \\
6-2 A_{s}-A_{t} \beta_{t}^{2}-3 \lambda+2 \Lambda & =0 \\
A_{s}-A_{t} \beta^{2}-\frac{3}{2 \lambda} A_{s} m^{2}+3 \lambda+2 \Lambda & =0 .
\end{aligned}
$$


Solving these four equations we express $m^{2}, A_{t}, A_{s}, \Lambda$ in terms of $\beta_{t}, \lambda$. The solution is

$$
\begin{aligned}
m^{2} & =-2 \lambda ; & A_{t} & =\frac{1+2 \beta_{t}^{2}}{\beta_{t}^{2}} ; \\
A_{s} & =1-\lambda ; & \Lambda & =\frac{1}{2}\left(2 \beta_{t}^{2}+\lambda-3\right) .
\end{aligned}
$$

Then, $A_{s} \geq 0, \Lambda>0, \beta_{t}>0$ implies

$$
\lambda \leq 1 ; \quad \beta_{t}>\sqrt{\frac{3-\lambda}{2}} .
$$

Finally, let us briefly also comment on the Type VIII case. ${ }^{11}$ Here the symmetry group is $\mathrm{SL}(2, R)$. An example would be the case already considered in section 4.2 in which case the spatial directions span $E A d S_{3}$ and the group of symmetries of the spatial manifold is enhanced to $\mathrm{SO}(3,1)$. However, we expect that more interesting examples, where the symmetry is only $\mathrm{SL}(2, R)$ symmetry and not enhanced, can also easily be found. These would be the analogues of the squashed $S^{3}$ example for the Type IX case above.

\section{An extremal brane interpolating from Type VII to $\boldsymbol{A d} \boldsymbol{S}_{5}$}

So far, our emphasis has been on finding solutions which have a scaling symmetry along with the generalised translational symmetries discussed above. One expects such scaling solutions to describe a dual field theory in the far infra-red and to arise as the nearhorizon limit of non-scale invariant solutions in general. An interesting feature of the field theory, suggested by the geometric description, is that in the IR it effectively lives in a curved spacetime. For example, consider the scaling solution of Type VII type which was described in section 3.1. One expects, roughly, that the spacetime seen by the dual field theory is given by a hypersurface at constant $r=r_{0}$ with $r_{0} \gg 1$. From eq. (3.7) we see that at $r=r_{0}$ this hypersurface is described by the metric,

$$
d s^{2}=R^{2}\left[-e^{2 \beta_{t} r_{0}} d t^{2}+\left(d x^{1}\right)^{2}+e^{2 \beta r_{0}}\left[\left(\omega^{2}\right)^{2}+\lambda^{2}\left(\omega^{3}\right)^{2}\right]\right] .
$$

From eq. (3.6) we see that this is a non-trivial curved spacetime.

This observation raises a question: can the scaling solutions of non-trivial Bianchi type, arise in situations where the field theory in the ultraviolet is in flat spacetime? Or do they require the field theory in the UV to itself be in a curved background? The latter case would be much less interesting from the point of view of possible connections with condensed matter systems. In the gravity description when the scaling solution arises from the near-horizon limit of a geometry which is asymptotically AdS or Lifshitz, this question takes the following form: is a normalizable deformation of the metric (or its analogue in

\footnotetext{
${ }^{11}$ In the Type IX case studied above the spatial directions span a squashed $S^{3}$ which is compact. In the Type VIII case the corresponding manifold is non-compact and is more likely to arise starting with an asymptotic geometry where the spatial directions are along $R^{3}$ with no non-normalizable metric perturbations.
} 
the Lifshitz case) enough? Or does one necessarily have to turn on a non-normalizable metric deformation to obtain the scaling solution of non-trivial Bianchi type in the IR?

In this section we will examine this question for the Type VII solution discussed in section 3.1. We will find, after enlarging the matter content to include two gauge fields, that normalizable deformations for the metric suffice. More specifically, in this example we will find solutions where the metric starts in the UV as being AdS space with a normalizable metric perturbation being turned on and ends in the IR as a scaling Type VII solution. However, the gauge fields do have a non-normalizable mode turned on in the UV. ${ }^{12}$ From the dual field theory point of view this corresponds to being in flat space but subjecting the system to a helically varying external current. In response, the gravity solution teaches us that the field theory flows to a fixed point in the infra-red with an "emergent" non-trivial metric of the Type VII kind.

The Type VII geometry breaks parity symmetry, as was discussed in section 3 . The Lagrangian for the example we consider preserves parity. In the solutions we find the parity violation is introduced by the non-normalizable deformations for the gauge fields, or in dual language, by the external current source.

In condensed matter physics there are often situations where the Hamiltonian itself breaks parity and the ground state of the system, in the absence of any external sources, has a helically varying order parameter. This happens for example in the cases discussed in $[3,4]$. It will be interesting to examine how such situations might arise in gravitational systems as well. In 5 dimensions a Chern-Simons term can be written for a gauge field and we expect that with parity violation incorporated in this way gravity solutions showing similar behaviour can also be easily found. For a discussion of helical type instabilities along these lines see [9-15].

A similar analysis, asking whether a non-trivial asymptotic metric along the spatial directions is needed in the first place, should also be carried out for the other Bianchi classes. As a first pass one can work in the thin wall approximation and ask whether the solution in the IR with the required homogeneity symmetry can be matched to AdS or a Lifshitz spacetime using a domain wall with a tension that meets reasonable energy conditions. We leave this for future work.

\subsection{The system and the essential idea}

We will consider a system consisting of gravity with a cosmological constant and two gauge fields $A_{1}, A_{2}$, with masses, $m_{1}, m_{2}$ described by the Lagrangian

$$
S=\int d^{5} x \sqrt{-g}\left\{R+\Lambda-\frac{1}{4} F_{1}^{2}-\frac{1}{4} F_{2}^{2}-\frac{1}{4} m_{1}^{2} A_{1}^{2}-\frac{1}{4} m_{2}^{2} A_{2}^{2}\right\} .
$$

We show that this system has a scaling solution of Type VII with parameters determined by $\Lambda, m_{1}^{2}, m_{2}^{2}$. This scaling solution will be the IR end of the full solution we will

\footnotetext{
${ }^{12}$ One of the gauge fields corresponds to a current which is a relevant operator in the IR and the other to an irrelevant operator. The current which breaks the symmetries of AdS to those of Type VII is relevant in the IR.
} 
construct which interpolates between the Type VII solution and asymptotic $A d S_{5}$ space in the UV.

The essential idea in constructing this full solution is as follows. It will turn out that the scaling solution, as one parameter is made small, becomes ${ }^{13} A d S_{2} \times R^{3}$. We will call this parameter $\lambda$ below. Now it is well known that $A d S_{2} \times R^{3}$ arises as the near-horizon geometry of an extremal RN brane which asymptotes to $A d S_{5}$ space. This means the $A d S_{2} \times R^{3}$ solution has a perturbation ${ }^{14}$ which grows in the UV and takes it to $A d S_{5}$ space. Since the Type VII solution we find is close to $A d S_{2} \times R^{3}$ when $\lambda$ is small, it is easy to identify the corresponding perturbation in this solution as well. Starting with the Type VII solution in the IR in the presence of this perturbation, we find, by numerically integrating the solution, that it asymptotes to $A d S_{5}$ in the UV as well with only a normalizable perturbation for the metric being turned on.

The Type VII solution, for small $\lambda$, is close to $A d S_{2} \times R^{3}$ in the following sense. At values of the radial coordinate which are not too big in magnitude the Type VII solution becomes approximately $A d S_{2} \times R^{3}$ with a small perturbation being turned on. This means that when one starts with the Type VII solution in the IR, with the perturbation that takes it to $A d S_{5}$ being now turned on, the solution first evolves to being close to $A d S_{2} \times R^{3}$ at moderate values of $r$ and then further evolves to $A d S_{5}$ in the UV.

Below we first describe the Type VII solution and the limit where it becomes $A d S_{2} \times R^{3}$. Then we describe the extreme RN geometry and its near horizon $A d S_{2} \times R^{3}$ limit and finally we identify the perturbation in the Type VII case and numerically integrate from the IR to UV showing that the geometry asymptotes to $A d S_{5}$.

\subsection{Type VII solutions}

Let us take the metric to be of the form

$$
d s^{2}=R^{2}\left\{d r^{2}-e^{2 \beta_{t} r} d t^{2}+\left(d x^{1}\right)^{2}+e^{2 \beta r}\left[\left(\omega^{2}\right)^{2}+(\lambda+1)\left(\omega^{3}\right)^{2}\right]\right\} .
$$

This metric is the same as in eq. (3.7) and has the same symmetries. The only difference is that the parameter $\lambda$ has been redefined. With our new definition when $\lambda \rightarrow 0$ the metric becomes of Lifshitz type. This follows from noting that $\left(\omega^{2}\right)^{2}+\left(\omega^{3}\right)^{2}=\left(d x^{2}\right)^{2}+\left(d x^{3}\right)^{2}$, eq. (3.6). In fact, once the equations of motion are imposed in this system, we will see that the $\lambda \rightarrow 0$ limit gives $A d S_{2} \times R^{3}$.

We take the gauge fields to have the form

$$
\begin{aligned}
& A_{2}=e^{\beta r} \sqrt{\tilde{A}_{c}} \omega^{2} \\
& A_{1}=e^{\beta_{t} r} \sqrt{\tilde{A}_{t}} d t .
\end{aligned}
$$

Note that the 6 variables, $R, \beta_{t}, \beta, \lambda, \tilde{A}_{c}, \tilde{A}_{t}$, are all constants and fully determine the solution. We will now solve the equations of motion to determine these constants in terms

\footnotetext{
${ }^{13}$ In the Type VII case the symmetries are generalised translations involving a combination of the usual translations and rotations along the spatial directions. In the $A d S_{2} \times R^{3}$ case these symmetries are enhanced to include both the usual translations and rotations along the spatial directions.

${ }^{14}$ These perturbations are given in eq. (5.47) and eq. (5.48) in section 5.3.
} 
of the parameters $\Lambda, m_{1}^{2}, m_{2}^{2}$, which enter in the action eq. (5.2). As in the discussion of section 3 it will be convenient to work in units where $R=1$.

The gauge field equations of motion then give

$$
\begin{aligned}
\sqrt{\tilde{A}_{t}}\left[4 \beta \beta_{t}-m_{1}^{2}\right] & =0 \\
\sqrt{\tilde{A}_{c}}\left[2 \beta\left(\beta_{t}+\beta\right)-\frac{2}{1+\lambda}-m_{2}^{2}\right] & =0 .
\end{aligned}
$$

The trace reversed Einstein equations with components along $t t, r r, x^{1} x^{1}$ directions give:

$$
\begin{aligned}
\frac{2 \tilde{A}_{c}}{1+\lambda}+2 \tilde{A}_{c} \beta^{2}+\tilde{A}_{t}\left(3 m_{1}^{2}+4 \beta_{t}^{2}\right)+4\left[\Lambda-3 \beta_{t}^{2}-6 \beta_{t} \beta\right] & =0 \\
\frac{\tilde{A}_{c}}{2(1+\lambda)}-\tilde{A}_{c} \beta^{2}+\beta_{t}^{2}\left(\tilde{A}_{t}-3\right)-6 \beta^{2}+\Lambda & =0 \\
\frac{3 \lambda^{2}+2 \tilde{A}_{c}}{1+\lambda}+\tilde{A}_{t} \beta_{t}^{2}-\tilde{A}_{c} \beta^{2}-2 \Lambda & =0 .
\end{aligned}
$$

While the components with indices along the $x^{2}, x^{3}$ directions lead to

$$
\begin{aligned}
\frac{6 \lambda(2+\lambda)-2 \tilde{A}_{c}}{1+\lambda}+3 m_{2}^{2} \tilde{A}_{c}+4 \beta\left(\tilde{A}_{c} \beta+3 \beta_{t}+6 \beta\right)+2 \tilde{A}_{t} \beta_{t}^{2}-4 \Lambda & =0 \\
\frac{3 \lambda(2+\lambda)-2 \tilde{A}_{c}}{1+\lambda}+\tilde{A}_{c} \beta^{2}-\tilde{A}_{t} \beta_{t}^{2}-6 \beta_{t} \beta-12 \beta^{2}+2 \Lambda & =0 .
\end{aligned}
$$

These are 7 equations in all, but one can check that only 6 of them are independent and these 6 independent equations determine the 6 parameters mentioned above. It turns out to be convenient to express the resulting solution as follows. Working in $R=1$ units, we express the parameters in the Lagrangian, $\Lambda$ and $m_{2}^{2}$, along with the constants in the solution $\beta_{t}, \beta, \tilde{A}_{t}, \tilde{A}_{c}$, in terms of $m_{1}^{2}$ and $\lambda$. This gives:

$$
\begin{aligned}
m_{2}^{2} & =-\frac{2}{1+\lambda}+\lambda(2-\epsilon \lambda) \\
\Lambda & =\frac{2}{\epsilon}+3 \epsilon \lambda^{2}+\lambda\left(-3+\frac{\lambda}{1-\lambda^{2}}\right) \\
\tilde{A}_{c} & =\frac{2 \lambda}{1-\lambda} \\
\tilde{A}_{t} & =\frac{4+\lambda(-4+\epsilon(5 \lambda-6))}{2(\lambda-1)(\epsilon \lambda-1)} \\
\beta_{t} & =\sqrt{\frac{2}{\epsilon}}(1-\epsilon \lambda) \\
\beta & =\sqrt{\frac{\epsilon}{2}} \lambda,
\end{aligned}
$$

where we have introduced a new parameter $\epsilon$ (not necessarily small) which is related to $m_{1}^{2}$ by

$$
m_{1}^{2}=4(1-\epsilon \lambda) \lambda
$$


Requiring $\tilde{A}_{t} \geq 0, \tilde{A}_{c} \geq 0$, and also requiring that $\beta_{t}, \beta$ have the same sign, leads to the conditions ${ }^{15}$

$$
0<\lambda<1, \quad 0<\epsilon \leq \frac{4}{\lambda} \frac{(1-\lambda)}{(6-5 \lambda)}
$$

We have chosen both $\beta_{t}>0, \beta \geq 0$, so that the horizon lies at $r \rightarrow-\infty$.

As in section 3 , we leave the important question about the stability of these solutions for future work.

\subsubsection{The limit}

Now consider the limit where $\lambda=0$. In this limit, we get

$$
\begin{array}{llll}
m_{2}^{2}=-2 & m_{1}^{2}=0 & \tilde{A}_{c}=0 & \beta=0 \\
\Lambda=\frac{2}{\epsilon} & \tilde{A}_{t}=2 & \beta_{t}=\sqrt{\frac{2}{\epsilon} .}
\end{array}
$$

It is easy to see that the resulting geometry is $A d S_{2} \times R^{3}$. It is sourced entirely by the gauge field $A_{1}$ which becomes massless. The second gauge field vanishes, since $\tilde{A}_{c}=0$.

Next consider a Type VII solution close to the limit $\lambda \rightarrow 0$, with $\epsilon$ being held fixed and of order unity. In this limit,

$$
\begin{aligned}
\beta_{t} & =\beta_{t}^{(0)}-\sqrt{2 \epsilon} \lambda \quad \text { where } \beta_{t}^{(0)}=\sqrt{\frac{2}{\epsilon}} \\
\beta & =\sqrt{\frac{\epsilon}{2}} \lambda \\
\tilde{A}_{t} & =2-\epsilon \lambda+\mathcal{O}\left(\lambda^{2}\right) \\
\tilde{A}_{c} & =2 \lambda+\mathcal{O}\left(\lambda^{2}\right) .
\end{aligned}
$$

Also we note that in this limit it follows from eq. (5.13), eq. (5.14), eq. (5.19) that

$$
\begin{aligned}
m_{2}^{2} & =-2+4 \lambda \\
\Lambda & =\frac{2}{\epsilon}-3 \lambda \\
m_{1}^{2} & =4 \lambda
\end{aligned}
$$

up to $O\left(\lambda^{2}\right)$ corrections.

Now consider an intermediate range for the radial variable where

$$
|r| \ll \frac{1}{\lambda} .
$$

In this intermediate region we can expand various components of the metric. E.g.,

$$
e^{2 \beta r} \simeq 1+2 \beta r
$$

\footnotetext{
${ }^{15}$ The equations allow for $\beta_{t}, \beta$ to have opposite sign, this would correspond to a strange dispersion relation for fluctuations though and probably would be unstable.
} 
etc. This gives for the metric

$$
\begin{aligned}
d s^{2} & =\left[d r^{2}-e^{2 \sqrt{\Lambda} r} d t^{2}+\left(d x^{1}\right)^{2}+\left(d x^{2}\right)^{2}+\left(d x^{3}\right)^{2}\right]+\delta d s^{2} \\
A_{1} & =\sqrt{2} e^{\sqrt{\Lambda} r} d t+\delta A_{1} \\
A_{2} & =\delta A_{2}
\end{aligned}
$$

where

$$
\begin{aligned}
\delta d s^{2} & =(\lambda r)\left[\sqrt{\frac{\epsilon}{2}} e^{2 \sqrt{\Lambda} r} d t^{2}+\sqrt{2 \epsilon}\left(\left(d x^{2}\right)^{2}+\left(d x^{3}\right)^{2}\right)\right]+\lambda\left(\omega^{3}\right)^{2} \\
\delta A_{1} & =-\left[\lambda r \frac{\sqrt{\epsilon}}{2}+\frac{\lambda \epsilon}{\sqrt{8}}\right] e^{\sqrt{\Lambda} r} d t \\
\delta A_{2} & =\sqrt{2 \lambda} \omega^{2}
\end{aligned}
$$

In obtaining these expressions we have used eq. (5.23) and eq. (5.28) to express $\beta_{t}^{(0)}$ in terms of $\Lambda$.

We see that when $\lambda \ll 1$ and eq. (5.30) is met the solution does reduce to $A d S_{2} \times R^{3}$ with only $A_{1}$ being turned on. There are additional perturbations in the metric and gauge fields, given by eq. (5.35), eq. (5.36), eq. (5.37). These are small in this region.

\subsection{Extremal RN brane}

In this subsection we turn off the gauge field $A_{2}$ and consider a system consisting of just gravity, with $\Lambda$, and $A_{1}$, with no mass. It is well known that this system has an extremal Reissner Nordstrom black brane solution with metric

$$
d s^{2}=-a(\tilde{r}) d t^{2}+\frac{1}{a(\tilde{r})} d \tilde{r}^{2}+b(\tilde{r})\left[d x^{2}+d y^{2}+d z^{2}\right]
$$

where

$$
a(\tilde{r})=\frac{\left(\tilde{r}-\tilde{r}_{h}\right)^{2}\left(\tilde{r}+\tilde{r}_{h}\right)^{2}\left(\tilde{r}^{2}+2 \tilde{r}_{h}^{2}\right) \Lambda}{12 \tilde{r}^{4}} ; \quad b(\tilde{r})=\tilde{r}^{2}
$$

and gauge field

$$
A=A_{t} d t
$$

where

$$
A_{t}(\tilde{r})=-\tilde{r}_{h} \sqrt{\frac{\Lambda}{2}}\left(\frac{\tilde{r}_{h}^{2}}{\tilde{r}^{2}}-1\right)
$$

The parameter $\tilde{r}_{h}$ determines the location of the horizon and is determined by the charge density carried by the extremal brane.

Near the horizon, as $\tilde{r} \rightarrow \tilde{r}_{h}$, the geometry becomes of $A d S_{2} \times R^{3}$ type. As discussed in appendix $\mathrm{D}$ by changing variables

$$
r=\int \frac{d \tilde{r}}{\sqrt{a(\tilde{r})}}
$$


one can express the metric and gauge fields for $\tilde{r} \rightarrow \tilde{r}_{h}$ as

$$
\begin{aligned}
d s^{2} & =d r^{2}-e^{2 \sqrt{\Lambda} r}\left(1-\frac{14}{3} e^{\sqrt{\Lambda} r}\right) d t^{2}+\left(1+2 e^{\sqrt{\Lambda} r}\right)\left(d x^{2}+d y^{2}+d z^{2}\right) \\
A_{t} & =\sqrt{2} e^{\sqrt{\Lambda} r}\left(1-\frac{8}{3} e^{\sqrt{\Lambda} r}+\cdots\right) d t
\end{aligned}
$$

It is useful to write these expressions as the leading terms

$$
\begin{aligned}
& d s^{2}=d r^{2}-e^{2 \sqrt{\Lambda} r} d t^{2}+\left(d x^{1}\right)^{2}+\left(d x^{2}\right)^{2}+\left(d x^{3}\right)^{2} \\
& A_{1 t}=\sqrt{2} e^{\sqrt{\Lambda} r}
\end{aligned}
$$

which correspond to $A d S_{2} \times R^{3}$ sourced by a gauge field in the time-like direction, and corrections,

$$
\begin{aligned}
& \delta d s^{2}=\frac{14}{3} e^{3 \sqrt{\Lambda} r} d t^{2}+2 e^{\sqrt{\Lambda} r}\left(\left(d x^{1}\right)^{2}+\left(d x^{2}\right)^{2}+\left(d x^{3}\right)^{2}\right) \\
& \delta A_{1 t}=-\frac{8}{3} \sqrt{2} e^{2 \sqrt{\Lambda} r}
\end{aligned}
$$

We see that the perturbations are small if

$$
e^{\sqrt{\Lambda} r} \ll 1
$$

which is the condition for being close enough to the horizon of the extremal brane. Note that this condition requires that $r$ be negative.

\subsection{The perturbation in Type VII}

We can now identify the perturbation in Type VII, with small $\lambda$, which becomes eq. (5.47), eq. (5.48) in the $\lambda \rightarrow 0$ limit. We write

$$
\begin{aligned}
d s^{2} & =d r^{2}-f(r) d t^{2}+g(r)\left(d x^{1}\right)^{2}+h(r)\left(\omega^{2}\right)^{2}+j(r)\left(\omega^{3}\right)^{2} \\
A_{1} & =A_{1 t}(r) d t \\
A_{2} & =A_{2}(r) \omega^{2}
\end{aligned}
$$

with

$$
\begin{aligned}
f(r) & =e^{2 \beta_{t} r}\left(1+\tilde{\epsilon} f_{c} e^{\nu r}\right) \\
g(r) & =1+\tilde{\epsilon} g_{c} e^{\nu r} \\
h(r) & =e^{2 \beta r}\left(1+\tilde{\epsilon} h_{c} e^{\nu r}\right) \\
j(r) & =e^{2 \beta r}(\lambda+1)\left(1+\tilde{\epsilon} j_{c} e^{\nu r}\right) \\
A_{1 t}(r) & =\sqrt{\tilde{A}_{t}} e^{\beta_{t} r}\left(1+\tilde{\epsilon} A_{1 c} e^{\nu r}\right) \\
A_{2}(r) & =\sqrt{\tilde{A}_{c}} e^{\beta r}\left(1+\tilde{\epsilon} A_{2 c} e^{\nu r}\right) .
\end{aligned}
$$


The parameters $\beta_{t}, \beta, A_{1 c}, A_{2 c}$ take values given in eq. (5.23), eq. (5.24), eq. (5.25), eq. (5.26), and $\tilde{\epsilon}$ is the small parameter which keeps the perturbation small.

It is easy to see that there is a solution to the resulting coupled linearized equations in which

$$
\nu=\sqrt{\frac{2}{\epsilon}}\left(1-\lambda \frac{20}{9} \epsilon\right) .
$$

By shifting $r$ we can always rescale $f_{c}$. For comparing with eq. (5.47), eq. (5.48) we take

$$
\tilde{\epsilon} f_{c}=-\frac{14}{3} \text {. }
$$

The other parameters then take the values

$$
\begin{aligned}
\tilde{\epsilon} g_{c} & =2\left(1+\lambda \frac{\epsilon}{378}(167+54 \epsilon)\right) \\
\tilde{\epsilon} h_{c} & =2\left(1+\lambda \frac{\epsilon(548-\epsilon(89+81 \epsilon))}{756(\epsilon-1)}\right) \\
\tilde{\epsilon} j_{c} & =2\left(1+\lambda \frac{\epsilon(170-\epsilon(467+81 \epsilon))}{756(\epsilon-1)}\right) \\
\tilde{\epsilon} A_{1 c} & =-\frac{8}{3}\left(1+\lambda \frac{(32-27 \epsilon) \epsilon}{2016}\right) \\
\tilde{\epsilon} A_{2 c} & =-\frac{1}{2} \epsilon+O(\lambda) .
\end{aligned}
$$

Note the $O(\lambda)$ correction to $A_{2 c}$ will contribute at higher order since $\tilde{A}_{c}$ is or order $\lambda$.

It is easy to see that as $\lambda \rightarrow 0$ this perturbation becomes exactly the one given in eq. (5.47), eq. (5.48).

\subsection{The interpolation}

We are finally ready to consider what happens if we start with the Type VII solution we have found in section 5.2 but now with the perturbation identified in the previous section being turned on. We work with the cases where $\lambda \ll 1$.

Since $\nu>0$ we see that as $r \rightarrow-\infty$ the effects of the perturbation becomes very small and the solutions becomes the Type VII solution discussed in section 5.2.

In the region where $r$ is negative and both conditions eq. (5.30) and eq. (5.49) are met we see that the resulting solution can be thought of as being approximately $A d S_{2} \times R^{3}$ with only $A_{1}$ being turned on along the time direction. There are corrections to this approximate solution given by the sum of the perturbations in eq. (5.35), eq. (5.36),eq. (5.37) and eq. (5.47), eq. (5.48).

To numerically integrate we will start in this region and then go further out to larger values of $r$. We take the metric and gauge fields to be given by eq. (5.50), eq. (5.51), eq. (5.52).

We are interested in a solution which asymptotes to $A d S_{5}$. It is best to work with values of $\epsilon$ for which $m_{2}^{2}$ lies above the $A d S_{5}$ BF bound. This condition gives $m_{2}^{2} / \Lambda>-\frac{1}{6}$. Working with the leading order terms in eq. (5.27), eq. (5.28) this condition is met if

$$
\epsilon<\frac{1}{6}
$$




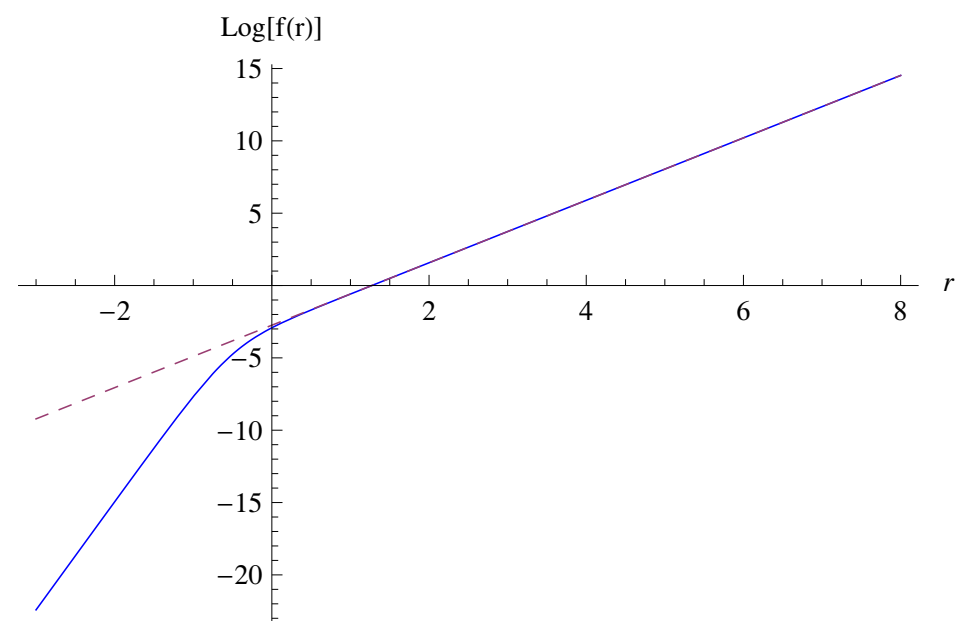

Figure 1. Numerical solution for metric component $\log f(r)$.

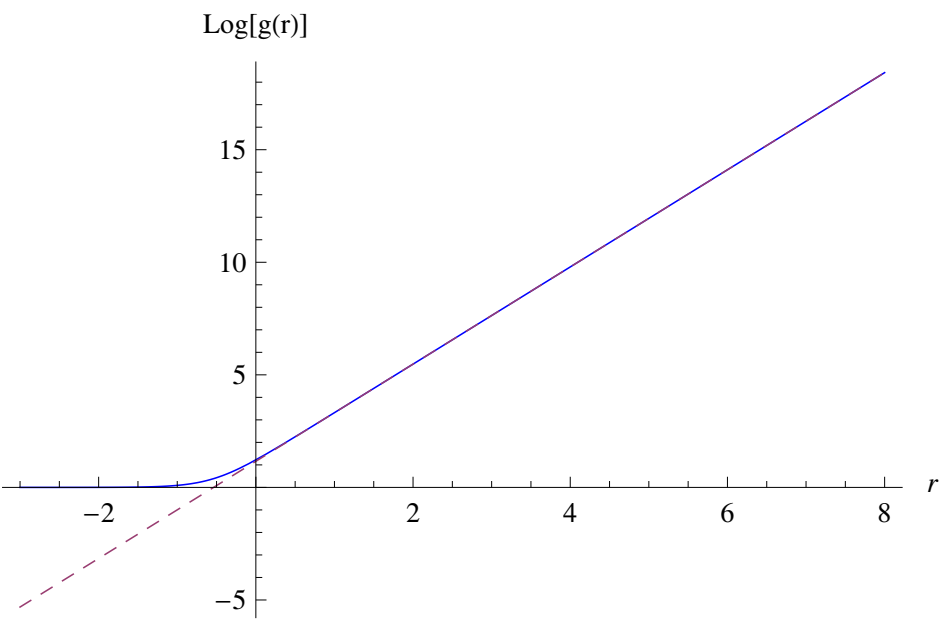

Figure 2. Numerical solution for metric component $\log g(r)$.

In the figures which are included below we have taken $\epsilon=\frac{1}{7}$. In addition, we take $\lambda=10^{-2}$. And we start the numerical integration at $r=-3$, which meets the conditions eq. (5.30), eq. (5.49). Varying these parameters, within a range of values, does not change our results in any essential way.

The reader will see from figures $1,2,3,4$, that the metric coefficients $f(r), g(r)$, $h(r), j(r)$, become exponential in $r$ as $r \rightarrow \infty$. We have fitted the slope and find agreement with the behavior in AdS space with cosmological constant given in eq. (5.28).

Notice that when $\lambda$ is small and positive the gauge field $A_{1}$ has a small and positive $m^{2}$, eq. (5.29). In asymptotic $A d S_{5}$ space such a gauge field has two solutions behaving like

$$
A_{1} \sim e^{\left(-\sqrt{\frac{\Lambda}{12}} \pm \sqrt{\frac{\Lambda}{12}+\frac{m_{1}^{2}}{2}}\right) r} .
$$




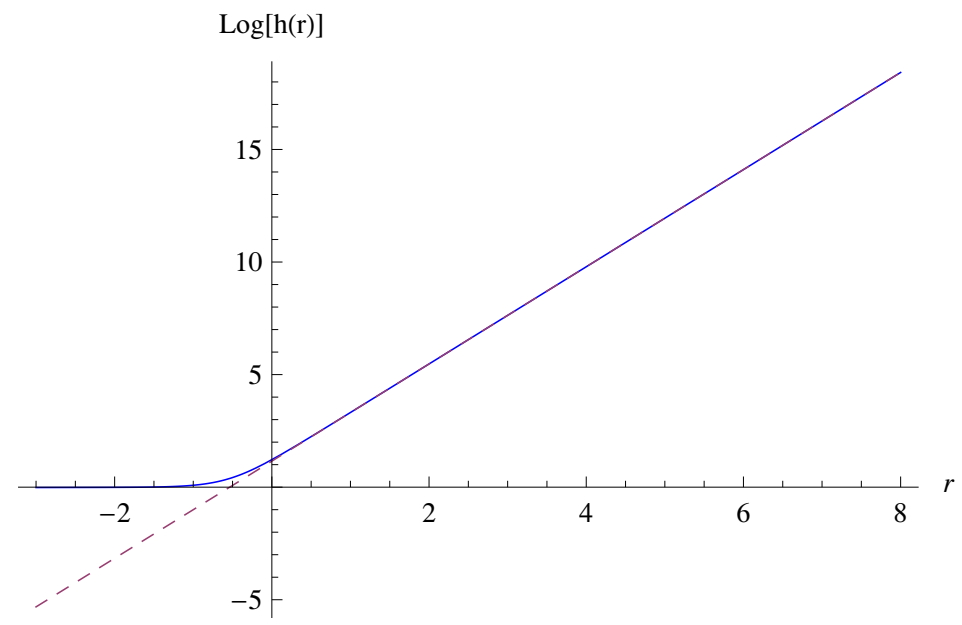

Figure 3. Numerical solution for metric component $\log h(r)$.

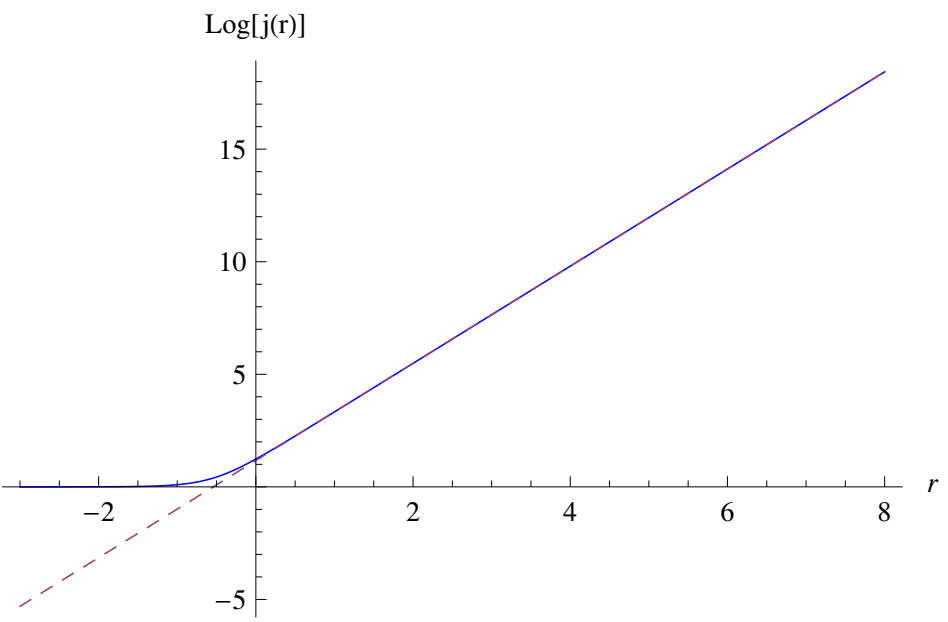

Figure 4. Numerical solution for metric component $\log j(r)$.

In particular one branch grows exponentially as $r \rightarrow \infty$. It is easy to see that this behavior agrees with the figure 5 at large $r$. The small value of $m_{1}^{2}$ means one has to go out to relatively large values of $r$ to see this behavior. In contrast, since $A_{2}$ has negative $m^{2}$ both the solutions asymptotically die out, which agrees with figure 6 .

One comment is worth making here. The reader might wonder why the asymptotically growing $A_{1}$ gauge field does not back react and lead to a departure from $A d S_{5}$ space. The reason is that $m_{1}^{2}$ is very slightly positive, i.e., $m_{1}^{2} / \Lambda \ll 1$, and as a result the stress energy due to this field is still subdominant compared to the cosmological term at large $r$.

In summary, the numerical integration shows that the resulting geometry is asymptotically $A d S_{5}$ with small corrections which correspond to normalizable perturbations being turned on. The gauge fields in contrast have both their normalizable and non-normalizable 


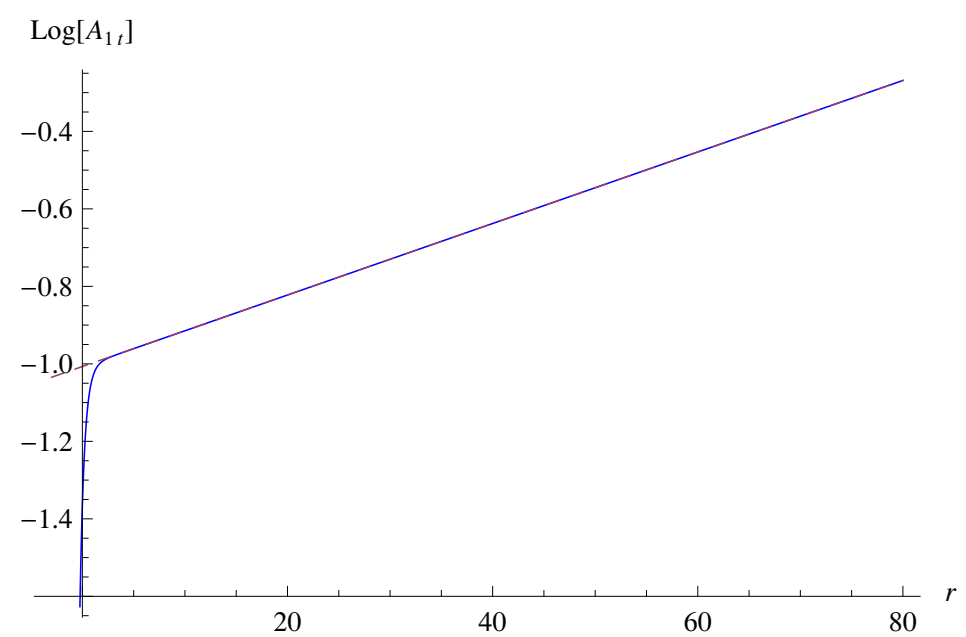

Figure 5. Numerical solution for gauge field $\log A_{1 t}(r)$.

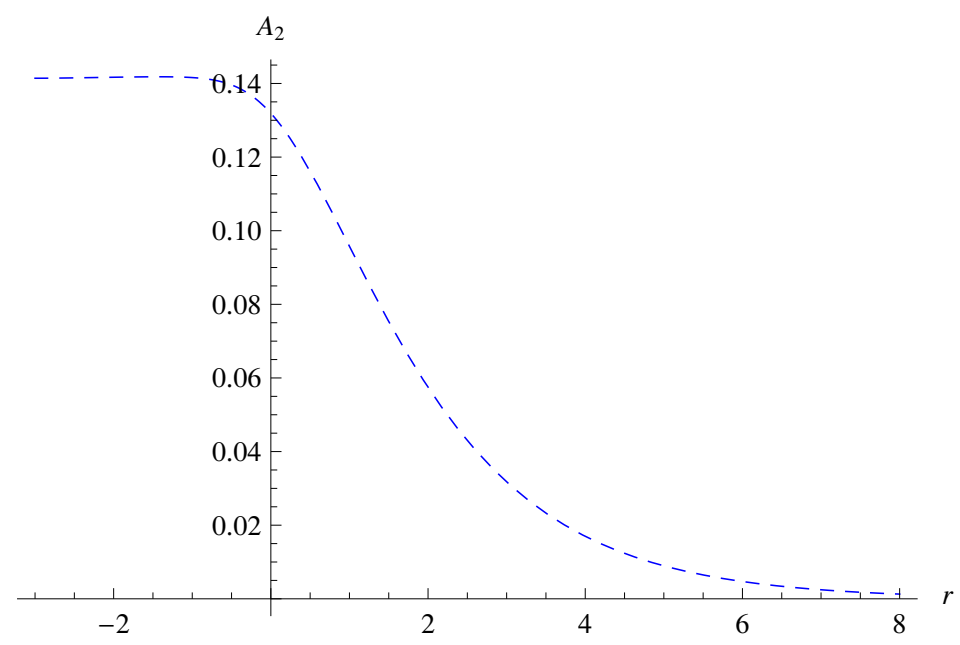

Figure 6. Numerical solution for gauge field $A_{2}(r)$.

modes turned on. This is clear in the $A_{1}$ case which grows exponentially, but it is also true for $A_{2}$ which has negative $m^{2}$ so that both modes for it die away asymptotically.

We have thus established that the Type VII geometry discussed in section 5.2 can arise starting from a flat boundary metric with non-normalizable gauge field deformations turned on.

\section{Generalised translations involving time and closed time-like curves}

So far, we have been discussing examples where the usual time translation symmetry is preserved and the generalised translations only involve the spatial directions. However, one can also have situations where the generalised translations involve the time direction in a 
non-trivial manner. For example, a case of Type $V I I_{0}$ type can arise when a translation in the time direction is accompanied by a rotation in a spatial two-plane. This could happen if there is a vector order parameter which is time dependent and rotates in the spatial twoplane as time advances. The time dependent order parameter might arise as a response to a suitable periodic time dependent external source.

We should note that a situation where time gets non-trivially involved in the generalised translations does not necessarily require the spacetime to be time dependent. For example it could have been that one has the standard translational symmetry along time and all the spatial directions except for one, $x^{1}$, and in the $x^{1}$ direction the translation is accompanied by a boost in the $t, x^{2}$ plane. In this case the metric would depend on $x^{1}$ but not $t$, just as in the case of Type VII discussed in section 3.1 the metric depended on $x^{1}$ but not $x^{2}, x^{3}$. Another example will be presented below where this point will also become clear.

Here, as in the discussion earlier, we will be interested in geometries which have scale invariance, along with the generalised translational symmetries mentioned above. Such gravitational solutions, from the point of view of possible connections with condensed matter physics, could describe dynamical critical phenomenon which arise when a system is subjected to time varying external forces.

In general, in the 9 Bianchi classes, the three generators of the symmetry algebra are inequivalent. Once we allow for the generalised translations to also involve time one typically finds that each Bianchi class gives rise to more than one physically distinct possibility depending on the role time translations play in the symmetry algebra.

We have not systematically examined which of the many resulting cases arising in this way can be realized by coupling reasonable kinds of matter fields to gravity, or even examined systematically which of these cases are allowed by the various energy conditions. These conditions could well impose fairly stringent restrictions in these cases where time is more non-trivially involved.

A few simple examples which we have constructed already illustrate some novel aspects which arise in such situations and we will limit ourselves to commenting on them here. In particular we will discuss below one example in which the resulting space-time may be physically unacceptable since it involves closed time-like curves. This example illustrates the need to proceed with extra caution in dealing with situations where the generalised translations also involve time.

The case we have in mind is most simply discussed in 4 total spacetime dimensions and is based on the Bianchi Type II algebra, which involves translations of a generalised sort along $t, x^{1}, x^{2}$, generated by Killing vectors

$$
\xi_{1}=\partial_{t}, \quad \xi_{2}=\partial_{1}, \quad \xi_{3}=\partial_{2}+x^{1} \partial_{t}
$$

The invariant one-forms are given by (see appendix A)

$$
\omega^{1}=d t-x^{2} d x^{1} ; \omega^{2}=d x^{1} ; \omega^{3}=d x^{2} .
$$

We assume the full metric to be also invariant under a translation in $r$ accompanied by a rescaling of $t, x^{1}, x^{2}$. Also we will take the metric to be diagonal in the basis of the invariant 
one-forms given above which gives ${ }^{16}$

$$
d s^{2}=R^{2}\left[d r^{2}-e^{2 \beta_{1} r}\left(d t-x^{2} d x^{1}\right)^{2}+e^{\beta_{1} r}\left(\left(d x^{1}\right)^{2}+\left(d x^{2}\right)^{2}\right)\right] .
$$

The gauge field is taken to be along the invariant one-form $\omega^{1}$ given in eq. (6.2) and of the form

$$
A=\sqrt{A_{1}} e^{\beta_{1} r} \omega^{1} .
$$

The gauge field equation of motion for this system gives

$$
m^{2}-2\left(1+\beta_{1}^{2}\right)=0
$$

The trace reversed Einstein equations along $r, t, x^{1}$ respectively are

$$
\begin{aligned}
A_{1}-6 \beta_{1}^{2}+A_{1} \beta_{1}^{2}+2 \Lambda & =0 \\
-2+A_{1}+A_{1} m^{2}-8 \beta_{1}^{2}+A_{1} \beta_{1}^{2}+2 \Lambda & =0 \\
2-A_{1}-4 \beta_{1}^{2}-A_{1} \beta_{1}^{2}+2 \Lambda & =0 .
\end{aligned}
$$

The Einstein equation along $x^{2}$ gives the same equation as along $x^{1}$.

It can be easily verified that the solution to these equations is

$$
m^{2}=2\left(1+\beta_{1}^{2}\right) \quad \Lambda=\frac{1}{2}\left(5 \beta_{1}^{2}-1\right) \quad A_{1}=1 .
$$

So we see that reasonable matter in the form of a massive Abelian gauge field, in the presence of a cosmological constant, can give rise to a geometry of Type II where time enters in a non-trivial way in the generalised translations.

However, as we will now see, this metric has closed time-like curves. This is a cause for physical concern, although similar solutions were investigated in [40] and suggestions about how CTCs may sometimes be acceptable features in such solutions were described there.

With a redefinition of the form,

$$
t-\frac{x^{1} x^{2}}{2} \rightarrow t \quad ; \quad x^{1} \rightarrow \rho \sin \theta \quad ; \quad x^{2} \rightarrow \rho \cos \theta
$$

the metric becomes

$$
d s^{2}=d r^{2}-e^{2 \beta_{1} r} d t^{2}+e^{\beta_{1} r} d \rho^{2}+e^{\beta_{1} r} \rho^{2}\left(1-\frac{\rho^{2}}{4} e^{\beta_{1} r}\right) d \theta^{2}+\rho^{2} e^{2 \beta_{1} r} d \theta d t .
$$

Here the coordinate $\theta$ is periodic with period $2 \pi$. Now notice that for $\left(1-\frac{\rho^{2}}{4} e^{\beta_{1} r}\right)<0, \theta$ becomes time-like and the closed curve, $\theta=[0,2 \pi]$, becomes a closed time-like curve. ${ }^{17}$

\footnotetext{
${ }^{16}$ Note that this metric is not time dependent, also note that for simplicity we are assuming that both spatial coordinates $x^{1}$ and $x^{2}$ scale similarly, but in general that is not required.

${ }^{17}$ This observation was made by R. Loganayagam. We thank him for some very helpful conversations.
} 


\section{Discussion}

In this paper, we argued that black branes need not be translationally invariant along the spatial directions in which they extend, and could instead have the less restrictive property of homogeneity. We showed that such black brane solutions in $4+1$ dimensions are classified by the Bianchi classification and fall into 9 classes. We mostly considered extremal black branes and focused on their near-horizon attractor region, which we took to be of scale invariant type. We found that such scale invariant solutions, realizing many of the non-trivial Bianchi classes, can arise in relatively simple gravitational systems with a negative cosmological constant and one or two massive Abelian gauge fields as matter. From the point of view of the holographically dual field theory, and of possible connections with condensed matter physics, these solutions correspond to new phases of matter which can arise when translational invariance is broken. ${ }^{18}$

It is clear that this paper has merely scratched the surface in exploring these new homogeneous brane solutions, and much more needs to be done to understand them better. Some directions for future work are as follows:

- One would like to analyze the stability properties of the near-horizon solutions we have found in more detail. In the examples we constructed, sometimes solutions in two different classes are allowed for the same range of parameters. For example, with one gauge field, both Lifshitz and Type VII can arise for the same mass range. In such cases especially one would like to know if both solutions are stable or if one of them has an instability.

- It seems reasonable to suggest that the scaling solutions we have obtained arise as the near-horizon limits of extremal black brane solutions. For the case of Type VII we showed that this was indeed true by obtaining a full extremal black brane solution which asymptotes to AdS space at large distance. It would be worthwhile to check if this is true for the other classes as well. ${ }^{19}$

- One would like to find the small temperature deformation of these solutions. This can be done working in the near-horizon limit itself. This is important in characterizing the thermodynamics of these systems better, and also in establishing that the solutions are the end points of non-extremal solutions. It is known that while curvature invariants are finite in the Lifshitz solutions, tidal forces do blow up (for a recent discussion of the nature of Lifshitz singularities, see e.g. [41]). We saw that this was also true in the Type VII case investigated in section 3.1.1, and expect it to be true in many solutions in the other Bianchi classes as well. It would be worth investigating this issue further. If a finite temperature deformation exists, it would probably allow us to control the effects due to these large tidal forces.

\footnotetext{
${ }^{18}$ Massive gauge fields in the bulk correspond to currents in the boundary theory which are not conserved. One expects that similar solutions should also arises when the symmetry is spontaneously broken in the bulk, corresponding to a superfluid in the boundary. It will be interesting to check if superfluids found in nature exhibit all of the phases we have found.

${ }^{19}$ In particular it is worth checking whether the asymptotic geometry along the spatial directions can be taken to be flat space for the other classes as well, as happens in the Type VII example we have constructed. We expect this to be true in several cases, for suitable matter content.
} 
- One should understand these solutions more deeply from the point of view of the attractor mechanism. The solutions are tightly constrained by symmetries, since they have the generalised translational invariances and scaling invariance (this involves a translation along the radial direction). These symmetries are enough to determine the solution completely up to a few constants, e.g. $R, \beta_{1}, \beta_{i}, A_{t}$ in eq. (3.3), eq. (3.5). It should be straightforward to formulate an entropy function for any given Bianchi class which can be extremised to obtain these constants, thereby determining the full solution from purely algebraic considerations. ${ }^{20}$ It would also be interesting to ask when a given scaling solution is an attractor in terms of varying the asymptotic data and studying how the solution changes, and to understand how to encode the attractor behavior (or lack thereof) in terms of suitable near-horizon data. We hope to report on this development soon.

- Going beyond solutions with scaling symmetry, one would like to incorporate the possibility that the near-horizon geometry does not have a scaling isometry, but instead has a conformal Killing vector corresponding to a shift of the radial direction and a rescaling of the other directions. Such metrics have been analyzed in [42-48] and shown to lead to interesting behaviours.

- It is quite amazing to us that even the relatively simple matter systems we have explored here, consisting of a just a couple of massive gauge fields, can yield such a large diversity of solutions. It would be fascinating to explore within a simple system of this type, which of the solutions are related to each other by RG flow.

- Naturally, one would like to know whether the kinds of simple gravitational systems we have analyzed here (with their required values of parameters) can arise in string theory. ${ }^{21}$ To be even more ambitious, one would ultimately like to ask about all of the phases that can be realized in gravitational systems which admit a consistent UV completion. The homogeneous phases we have investigated here are many more than were previously considered, but one suspects that even they are only a tiny subset of all possibilities!

Clearly, in gravity, as in condensed matter physics, more is different and there is much left to be done.

\section{Acknowledgments}

We thank Gary Horowitz, Renata Kallosh, R. Loganayagam, Kengo Maeda, Gautam Mandal, Shiraz Minwalla, Mike Mulligan, Eric Perlmutter, Shiroman Prakash, Ramadas Ramakrishnan and especially Kedar Damle and Ashoke Sen for helpful discussions. NI, SK and SPT thank the organizers of the meeting on "Hot Nuclei, Cold Atoms and Black

\footnotetext{
${ }^{20}$ We are thankful to Ashoke Sen for very helpful discussions in this regard.

${ }^{21}$ Some brane solutions of supergravity theories with instabilities which break translational invariance have been identified in the literature [10-15]. It would also be interesting to ask whether the attractor solutions we have found describe the end points for these instabilities.
} 
Holes" held at the ASICTP, Trieste. SPT also thanks the organizers of the Higher Spin Field Theories meeting held in HRI, Allahabad, and NK, PN, NS and SPT thank the organizers of the National Strings Meeting held Delhi, for providing a stimulating atmosphere where some of this research was carried out. NI would like to thank RIKEN for their kind hospitality where part of this work was done. SK acknowledges the Aspen Center for Physics for hospitality while this work was in progress, and thanks the organizers of the "Holographic Duality and Condensed Matter Physics" workshop at the KITP for providing such a stimulating atmosphere. NK, PN, NS and SPT acknowledge funding from the Government of India, and thank the people of India for generously supporting research in string theory. SK was supported by the US DOE under contract DE-AC02-76SF00515 and the National Science Foundation under grant no. PHY-0756174.

\section{A Three dimensional homogeneous spaces}

A three dimensional homogeneous space has three linearly independent Killing vector fields, $\xi_{i}, i=1,2,3$. The infinitesimal transformations generated by these Killing vectors take any point in the space to any other point in its immediate vicinity. The Killing vectors satisfy a three dimensional real algebra with commutation relations

$$
\left[\xi_{i}, \xi_{j}\right]=C_{i j}^{k} \xi_{k} .
$$

As discussed in $[1,2]$ there are 9 different such algebras, up to basis redefinitions, and thus 9 different classes of such homogeneous spaces. This classification is called the Bianchi classification and the 9 classes are the 9 Bianchi classes.

Also, as is discussed in $[1,2]$, in each case there are three linearly independent invariant vector fields, $X_{i}$, which commute with the three Killing vectors

$$
\left[\xi_{i}, X_{j}\right]=0
$$

The $X_{i}$ 's in turn satisfy the algebra

$$
\left[X_{i}, X_{j}\right]=-C_{i j}^{k} X_{k}
$$

There are also three one-forms, $\omega^{i}$, which are dual to the invariant vectors $X_{i}$. The Lie derivatives of these one-forms along the $\xi_{i}$ directions also vanish making them invariant along the $\xi$ directions as well. The $\omega^{i}$ 's satisfy the relations

$$
d \omega^{i}=\frac{1}{2} C_{j k}^{i} \omega^{j} \wedge \omega^{k}
$$

Below we give a list which contains the structure constants for the 9 Bianchi algebras, in a particular basis of generators. For all the classes that arise in this paper we also give the Killing vector fields, invariant one-forms and invariant vector fields, in a particular coordinate basis. See [2] for more details.

- Type I: $C_{j k}^{i}=0$

$$
\xi_{i}=X_{i}=\partial_{i}, \omega^{i}=d x^{i}, d \omega^{i}=0
$$


- Type II: $C_{23}^{1}=-C_{32}^{1}=1$ and rest $C_{j, k}^{i}=0$

$$
\begin{array}{llll}
\xi_{1}=\partial_{2} & X_{1}=\partial_{2} & \omega^{1}=d x^{2}-x^{1} d x^{3} & d \omega^{1}=\omega^{2} \wedge \omega^{3} \\
\xi_{2}=\partial_{3} & X_{2}=x^{1} \partial_{2}+\partial_{3} & \omega^{2}=d x^{3} & d \omega^{2}=0 \\
\xi_{3}=\partial_{1}+x^{3} \partial_{2} & X_{3}=\partial_{1} & \omega^{3}=d x^{1} & d \omega^{3}=0
\end{array}
$$

- Type III: $C_{13}^{1}=-C_{31}^{1}=1$ and rest $C_{j, k}^{i}=0$

$$
\begin{array}{llll}
\xi_{1}=\partial_{2} & X_{1}=e^{x^{1}} \partial_{2} & \omega^{1}=e^{-x^{1}} d x^{2} & d \omega^{1}=\omega^{1} \wedge \omega^{3} \\
\xi_{2}=\partial_{3} & X_{2}=\partial_{3} & \omega^{2}=d x^{3} & d \omega^{2}=0 \\
\xi_{3}=\partial_{1}+x^{2} \partial_{2} & X_{3}=\partial_{1} & \omega^{3}=d x^{1} & d \omega^{3}=0
\end{array}
$$

- Type V: $C_{13}^{1}=-C_{31}^{1}=1, C_{23}^{2}=-C_{32}^{2}=1$ and rest $C_{j, k}^{i}=0$

$$
\begin{array}{llll}
\xi_{1}=\partial_{2} & X_{1}=e^{x^{1}} \partial_{2} & \omega^{1}=e^{-x^{1}} d x^{2} & d \omega^{1}=\omega^{1} \wedge \omega^{3} \\
\xi_{2}=\partial_{3} & X_{2}=e^{x^{1}} \partial_{3} & \omega^{2}=e^{-x^{1}} d x^{3} & d \omega^{2}=\omega^{2} \wedge \omega^{3} \\
\xi_{3}=\partial_{1}+x^{2} \partial_{2}+x^{3} \partial_{3} & X_{3}=\partial_{1} & \omega^{3}=d x^{1} & d \omega^{3}=0
\end{array}
$$

- Type VI: $C_{13}^{1}=-C_{31}^{1}=1, C_{23}^{2}=-C_{32}^{2}=h$ with $(h \neq 0,1)$ and rest $C_{j, k}^{i}=0$

$$
\begin{array}{llll}
\xi_{1}=\partial_{2} & X_{1}=e^{x^{1}} \partial_{2} & \omega^{1}=e^{-x^{1}} d x^{2} & d \omega^{1}=\omega^{1} \wedge \omega^{3} \\
\xi_{2}=\partial_{3} & X_{2}=e^{h x^{1}} \partial_{3} & \omega^{2}=e^{-h x^{1}} d x^{3} & d \omega^{2}=h \omega^{2} \wedge \omega^{3} \\
\xi_{3}=\partial_{1}+x^{2} \partial_{2}+h x^{3} \partial_{3} & X_{3}=\partial_{1} & \omega^{3}=d x^{1} & d \omega^{3}=0
\end{array}
$$

- Type VII $\mathbf{V}_{0} C_{23}^{1}=-C_{32}^{1}=-1, C_{13}^{2}=-C_{31}^{2}=1$ and rest $C_{j, k}^{i}=0$

$$
\begin{array}{ll}
\xi_{1}=\partial_{2} & X_{1}=\cos \left(x^{1}\right) \partial_{2}+\sin \left(x^{1}\right) \partial_{3} \\
\xi_{2}=\partial_{3} & X_{2}=-\sin \left(x^{1}\right) \partial_{2}+\cos \left(x^{1}\right) \partial_{3} \\
\xi_{3}=\partial_{1}-x^{3} \partial_{2}+x^{2} \partial_{3} & X_{3}=\partial_{1}
\end{array}
$$

And also,

$$
\begin{aligned}
\omega^{1} & =\cos \left(x^{1}\right) d x^{2}+\sin \left(x^{1}\right) d x^{3} & & d \omega^{1}=-\omega^{2} \wedge \omega^{3} \\
\omega^{2} & =-\sin \left(x^{1}\right) d x^{2}+\cos \left(x^{1}\right) d x^{3} & & d \omega^{2}=\omega^{1} \wedge \omega^{3} \\
\omega^{3} & =d x^{1} & & d \omega^{3}=0
\end{aligned}
$$

- Type IX: $C_{23}^{1}=-C_{32}^{1}=1, C_{31}^{2}=-C_{13}^{2}=1, C_{12}^{3}=-C_{21}^{3}=1$ and rest are zero

$$
\begin{aligned}
& \xi_{1}=\partial_{2} \\
& \xi_{2}=\cos \left(x^{2}\right) \partial_{1}-\cot \left(x^{1}\right) \sin \left(x^{2}\right) \partial_{2}+\frac{\sin \left(x^{2}\right)}{\sin \left(x^{1}\right)} \partial_{3} \\
& \xi_{3}=-\sin \left(x^{2}\right) \partial_{1}-\cot \left(x^{1}\right) \cos \left(x^{2}\right) \partial_{2}+\frac{\cos \left(x^{2}\right)}{\sin \left(x^{1}\right)} \partial_{3}
\end{aligned}
$$


With

$$
\begin{aligned}
& X_{1}=-\sin \left(x^{3}\right) \partial_{1}+\frac{\cos \left(x^{3}\right)}{\sin \left(x^{1}\right)} \partial_{2}-\cot \left(x^{1}\right) \cos \left(x^{3}\right) \partial_{3} \\
& X_{2}=\cos \left(x^{3}\right) \partial_{1}+\frac{\sin \left(x^{3}\right)}{\sin \left(x^{1}\right)} \partial_{2}-\cot \left(x^{1}\right) \sin \left(x^{3}\right) \partial_{3} \\
& X_{3}=\partial_{3}
\end{aligned}
$$

And also,

$$
\begin{aligned}
\omega^{1} & =-\sin \left(x^{3}\right) d x^{1}+\sin \left(x^{1}\right) \cos \left(x^{3}\right) d x^{2} ; & & d \omega^{1}=\omega^{2} \wedge \omega^{3} \\
\omega^{2} & =\cos \left(x^{3}\right) d x^{1}+\sin \left(x^{1}\right) \sin \left(x^{3}\right) d x^{2} ; & & d \omega^{2}=\omega^{3} \wedge \omega^{1} \\
\omega^{3} & =\cos \left(x^{1}\right) d x^{2}+d x^{3} ; & & d \omega^{3}=\omega^{1} \wedge \omega^{2}
\end{aligned}
$$

For Types IV and VIII we give the structure constants only. For more explicit data on these Types, see [2]

- Type IV: $C_{13}^{1}=-C_{31}^{1}=1, C_{23}^{1}=-C_{32}^{1}=1, C_{23}^{2}=-C_{32}^{2}=1$ and rest $C_{j, k}^{i}=0$

- Type $\mathbf{V I I}_{\mathbf{h}}\left(\mathbf{0}<\mathbf{h}^{2}<\mathbf{4}\right): C_{13}^{2}=-C_{31}^{2}=1, C_{23}^{1}=-C_{32}^{1}=-1, C_{23}^{2}=-C_{32}^{2}=h$ and rest $C_{j, k}^{i}=0$

- Type VIII: $C_{23}^{1}=-C_{32}^{1}=-1, C_{31}^{2}=-C_{13}^{2}=1, C_{12}^{3}=-C_{21}^{3}=1$ and rest $C_{j, k}^{i}=0$

\section{B Gauge field equation of motion}

In this appendix we will consider a system with action eq. (3.4) and metric of form

$$
d s^{2}=d r^{2}-e^{2 \beta_{t} r} d t^{2}+\eta_{i j}(r) \omega^{i} \omega^{j}
$$

and derive the equation of motion for the gauge field. In eq. (B.1) $\omega^{i}, i=1,2,3$, are the three invariant one-forms along the spatial directions in which the brane extends.

To preserve the generalised translation symmetries along the spatial directions the gauge potential must take the form

$$
A=\sum_{i} f_{i}(r) \omega^{i}+A_{t}(r) d t
$$

Eventually, we will take $\eta_{i j}$ to be diagonal

$$
\eta_{i j}=\left(\lambda_{1}^{2} e^{2 \beta_{1} r}, \lambda_{2}^{2} e^{2 \beta_{2} r}, \lambda_{3}^{2} e^{2 \beta_{3} r}\right)
$$

and the functions appearing in the gauge field to be of the form

$$
f_{i}(r)=\tilde{A}_{i} e^{\beta_{i} r}, \quad A_{t}(r)=A_{t} e^{\beta_{t} r} .
$$

where $\lambda_{i}, \beta_{i}, \beta_{t}, \tilde{A}_{i}, A_{t}$ are all constants independent of all coordinates. For now though, we keep them to be general and proceed. 
The gauge field equation of motion is

$$
d *_{5} F=-\frac{1}{2} m^{2} *_{5} A .
$$

Only two cases are relevant for the discussion above. Either the gauge field has components only along the spatial directions or only along time. We discuss them in turn below.

\section{B.1 Gauge field with components along spatial directions}

Using $d \omega^{i}=\frac{1}{2} C^{i}{ }_{j k} \omega^{j} \wedge \omega^{k}$, we get the field strength to be ${ }^{22}$

$$
F=d A=f_{i}^{\prime}(r) d r \wedge \omega^{i}+\frac{1}{2} f_{i}(r) C_{j k}^{i} \omega^{j} \wedge \omega^{k} .
$$

With a choice of orientation so that in the basis $\left(\omega^{1}, \omega^{2}, \omega^{3}, d r, d t\right), \epsilon_{123 r t}>0$, we have the following Hodge dualities:

$$
\begin{aligned}
*_{5} \omega^{i} & =\frac{e^{\beta_{t} r}}{2} \sqrt{\eta} \eta^{i j} \epsilon_{j k l} \omega^{k} \wedge \omega^{l} \wedge d r \wedge d t \\
*_{5}\left(\omega^{j} \wedge \omega^{k}\right) & =\frac{e^{\beta_{t} r}}{\sqrt{\eta}} \eta_{i l} \epsilon^{j k l} \omega^{i} \wedge d r \wedge d t \\
*_{5}\left(d r \wedge \omega^{i}\right) & =-\frac{\sqrt{\eta} e^{\beta_{t} r}}{2} \eta^{i j} \epsilon_{j k l} \omega^{k} \wedge \omega^{l} \wedge d t .
\end{aligned}
$$

Here we are using notation such that $\epsilon^{i j k}=\epsilon_{i j k}=1$.

Thus the R.H.S of the gauge field equation, eq (B.5), becomes,

$$
-\frac{1}{2} m^{2} *_{5} A=-\frac{1}{4} m^{2} f_{i}(r) \eta^{i j} \sqrt{\eta} e^{\beta_{t} r} \epsilon_{j n p} \omega^{n} \wedge \omega^{p} \wedge d r \wedge d t
$$

The L.H.S of the gauge field equation, eq (B.5), becomes,

$$
\begin{aligned}
d *_{5} F= & d\left(-\frac{\sqrt{\eta} e^{\beta_{t} r}}{2} f_{i}^{\prime}(r) \eta^{i j} \epsilon_{j k l} \omega^{k} \wedge \omega^{l} \wedge d t+\frac{e^{\beta_{t} r}}{2 \sqrt{\eta}} f_{i}(r) C_{j k}^{i} \eta_{l e} \epsilon^{e j k} \omega^{l} \wedge d r \wedge d t\right) \\
= & {\left[-\frac{1}{2}\left(f_{i}^{\prime}(r) e^{\beta_{t} r} \sqrt{\eta} \eta^{i j}\right)^{\prime} \epsilon_{j n p}+\frac{1}{4 \sqrt{\eta}} f_{i}(r) e^{\beta_{t} r} C_{j k}^{i} \eta_{l e} C^{l}{ }_{n p} \epsilon^{e j k}\right] \omega^{n} \wedge \omega^{p} \wedge d r \wedge d t } \\
& -\frac{1}{2} f_{i}^{\prime}(r) e^{\beta_{t} r} \sqrt{\eta} \eta^{i j} \epsilon_{j k l} C_{q n}^{k} \omega^{q} \wedge \omega^{n} \wedge \omega^{l} \wedge d t .
\end{aligned}
$$

Comparing eq. (B.10), eq. (B.11) we see that

$$
f_{i}^{\prime}(r) e^{\beta_{t} r} \sqrt{\eta} \eta^{i j} \epsilon_{j k l} C_{q n}^{k} \epsilon^{q n l}=0 .
$$

Defining

$$
\begin{aligned}
\epsilon^{i j l} C^{k}{ }_{i j} & \equiv 2 C^{l k} \\
\epsilon_{i j k} C^{i j} & \equiv 2 a_{k}
\end{aligned}
$$

\footnotetext{
${ }^{22}$ Note in our convention $F=\frac{1}{2} F_{\mu \nu} d x^{\mu} \wedge d x^{\nu}$.
} 
as in [1], we get the condition that

$$
\text { either } \quad a_{k}=0 \quad \text { or } \quad f_{i}^{\prime}(r) e^{\beta_{t} r} \sqrt{\eta} \eta^{i j}(r) a_{j}=0 .
$$

Here we consider the case when $a_{k}=0$, this includes Type I, II, III, VII 0 , VIII, IX. In particular it covers all cases discussed in the main text where $A$ is oriented along the spatial directions.

Now comparing the $\omega \wedge \omega \wedge d r \wedge d t$ terms in eq. (B.10), eq. (B.11) and multiplying by $\epsilon^{m n p}$ on both sides we get

$$
\frac{1}{2} m^{2} \sqrt{\eta} f_{i}(r) e^{\beta_{t} r} \eta^{i m}=\left(f_{i}^{\prime}(r) e^{\beta_{t} r} \sqrt{\eta} \eta^{i m}\right)^{\prime}-\frac{e^{\beta_{t} r}}{\sqrt{\eta}} f_{i}(r) C^{j i} C^{m l} \eta_{l j} .
$$

To proceed let us consider the metric to be of form eq. (B.3). Also note that the gauge field is assumed to be of form as in eq. (B.4) but with $A_{t}=0$. Also we take $C^{j i}$ to be diagonal of form

$$
C^{j i}=\delta^{j i} k^{j} \quad(\text { with no sum over the index } j),
$$

for some constants $k^{j}$ such that $2 a_{k}=\epsilon_{i j k} C^{i j}=0$. This is equivalent to

$$
\left.C^{i}{ }_{j k}=\epsilon_{i j k} k^{i} \quad \text { (with no sum over the index } i\right) \text {. }
$$

Then eq. (B.16) says that for every value of the index $i=1,2,3$, such that $\tilde{A}_{i}$ is nonvanishing, following two conditions must be met:

$$
\sum_{j} \beta_{j}=2 \beta_{i}
$$

and

$$
\left[\frac{1}{2} m^{2}-\beta_{i}\left(-\beta_{i}+\beta_{t}+\sum_{j} \beta_{j}\right)\right]=-\frac{\lambda_{i}^{4}}{\tilde{\lambda}^{2}}\left(k^{i}\right)^{2}
$$

where $\tilde{\lambda}=\lambda_{1} \lambda_{2} \lambda_{3}$.

\section{B.2 Gauge field with components only along time}

Next take the case where the gauge field has only a component along the time direction,

$$
A=A_{t}(r) d t
$$

The field strength becomes

$$
F=d A=A_{t}^{\prime}(r) d r \wedge d t
$$

Using the Hodge star relations,

$$
\begin{aligned}
*_{5}(d r \wedge d t) & =-\frac{\sqrt{\eta} e^{-\beta_{t} r}}{6} \epsilon_{i j k} \omega^{i} \wedge \omega^{j} \wedge \omega^{k} \\
*_{5}(d t) & =\frac{\sqrt{\eta} e^{-\beta_{t} r}}{6} \epsilon_{i j k} d r \wedge \omega^{i} \wedge \omega^{j} \wedge \omega^{k},
\end{aligned}
$$


we get

$$
\begin{aligned}
d *_{5} F & =-\frac{\epsilon_{i j k}}{6}\left(\sqrt{\eta} e^{-\beta_{t} r} A_{t}^{\prime}(r)\right)^{\prime} d r \wedge \omega^{i} \wedge \omega^{j} \wedge \omega^{k} \\
*_{5} A & =A_{t}(r) \frac{\sqrt{\eta} e^{-\beta_{t} r}}{6} \epsilon_{i j k} d r \wedge \omega^{i} \wedge \omega^{j} \wedge \omega^{k} .
\end{aligned}
$$

Thus the gauge field equation of motion eq. (B.5) becomes

$$
\left(\sqrt{\eta} e^{-\beta_{t} r} A_{t}^{\prime}(r)\right)^{\prime}=\frac{m^{2}}{2} A_{t}(r) \sqrt{\eta} e^{-\beta_{t} r} .
$$

With a metric eq. (B.3) and gauge field

$$
A_{t}(r)=A_{t} e^{\beta_{t} r},
$$

we get

$$
2 \beta_{t} \sum_{i} \beta_{i}=m^{2}
$$

where $\tilde{\lambda}=\lambda_{1} \lambda_{2} \lambda_{3}$.

\section{Lifshitz solutions}

In this appendix we examine Lifshitz solutions which are known to arise in the system, eq. (3.4). Let us consider an ansatz,

$$
d s^{2}=d r^{2}-e^{2 \beta_{t} r} d t^{2}+e^{2 \beta_{i} r}\left(d x^{i}\right)^{2}
$$

where $i=1,2,3$. Let us first turn on the gauge field along time direction,

$$
A=\sqrt{A_{t}} e^{\beta_{t} r} d t .
$$

The Maxwell equation gives

$$
m^{2}=2 \beta_{t}\left(\beta_{1}+\beta_{2}+\beta_{3}\right)
$$

and the trace reversed Einstein equations give

$$
\begin{aligned}
A_{t} \beta_{t}^{2}-3\left(\beta_{t}^{2}+\beta_{1}^{2}+\beta_{2}^{2}+\beta_{3}^{2}\right)+\Lambda & =0 \\
A_{t}\left(3 m^{2}+4 \beta_{t}^{2}\right)-12 \beta_{t}\left(\beta_{t}+\beta_{1}+\beta_{2}+\beta_{3}\right)+4 \Lambda & =0 \\
A_{t} \beta_{t}^{2}+6 \beta_{1}\left(\beta_{t}+\beta_{1}+\beta_{2}+\beta_{3}\right)-2 \Lambda & =0 \\
A_{t} \beta_{t}^{2}+6 \beta_{2}\left(\beta_{t}+\beta_{1}+\beta_{2}+\beta_{3}\right)-2 \Lambda & =0 \\
A_{t} \beta_{t}^{2}+6 \beta_{3}\left(\beta_{t}+\beta_{1}+\beta_{2}+\beta_{3}\right)-2 \Lambda & =0 .
\end{aligned}
$$

The last three equations show $\beta_{i}=\beta$ for all $i$. Using this in the Maxwell equation we get

$$
m^{2}=6 \beta \beta_{t}
$$

Then solving the other equation of motion gives the solution as

$$
\beta_{i}=\beta \forall i
$$




$$
\begin{aligned}
m^{2} & =6 \beta \beta_{t} \\
\Lambda & =\beta_{t}^{2}+2 \beta \beta_{t}+9 \beta^{2} \\
A_{t} & =2\left(1-\frac{\beta}{\beta_{t}}\right) .
\end{aligned}
$$

If $\beta_{t}>0, \beta_{i}>0$,then $m^{2}$ is always positive. For the gauge field to be real, $\beta_{t}>\beta$.

Now, with the same metric ansatz, we choose the gauge field to be oriented along any one of $x^{i}$ directions. Without loss of generality, let us choose it to be oriented along $x^{1}$ :

$$
A=\sqrt{A_{1}} e^{\beta_{1} r} d x^{1} .
$$

The equation of motion can be obtained from the previous case by $\beta_{t} \leftrightarrow \beta_{1}$ and $A_{t} \rightarrow-A_{1}$. Then solving the equation of motion gives

$$
\begin{aligned}
\beta_{t}=\beta_{i} & =\beta \forall i \neq 1 \\
m^{2} & =6 \beta \beta_{1} \\
\Lambda & =\beta_{1}^{2}+2 \beta \beta_{1}+9 \beta^{2} \\
A_{1} & =2\left(\frac{\beta}{\beta_{1}}-1\right) .
\end{aligned}
$$

Again, if $\beta_{t}>0, \beta_{i}>0$, then $m^{2}$ is always positive. For the gauge field to be real $\beta>\beta_{1}$.

\section{Extremal RN solution}

Starting with the action eq. (3.4), and setting $m^{2}=0$, one gets the well known Reissner Nordstrom black brane solution.

It has the metric

$$
d s^{2}=-a(\tilde{r}) d t^{2}+\frac{1}{a(\tilde{r})} d \tilde{r}^{2}+b(\tilde{r})\left[d x^{2}+d y^{2}+d z^{2}\right]
$$

with

$$
a(\tilde{r})=\frac{Q^{2}}{12 \tilde{r}^{4}}+\frac{\tilde{r}^{2} \Lambda}{12}-\frac{M}{\tilde{r}^{2}} \quad b(\tilde{r})=\tilde{r}^{2}
$$

and the gauge field,

$$
A=-\left(\frac{Q}{2 \tilde{r}^{2}}-\frac{Q}{2 \tilde{r}_{h}^{2}}\right) d t
$$

Here $Q$ is charge and $M$ is the mass of the black brane. In the extremal limit we get $a^{\prime}\left(\tilde{r}_{h}\right)=0$ and also $a\left(\tilde{r}_{h}\right)=0$. This allows us to solve for $Q$ and $M$ in terms of $\tilde{r}_{h}$,

$$
\begin{aligned}
M & =\frac{\tilde{r}_{h}^{4} \Lambda}{4} \\
Q^{2} & =2 \tilde{r}_{h}^{6} \Lambda,
\end{aligned}
$$


giving eq. (5.39), eq. (5.41).

Now consider a new radial coordinate, $\tilde{r}$, given by eq. (5.42). The relation between the coordinates near $\tilde{r}=\tilde{r}_{h}$ is given by

$$
\sqrt{\Lambda} r=\log \left(\frac{\tilde{r}-\tilde{r}_{h}}{\tilde{r}_{h}}\right)+\frac{7}{6} \frac{\tilde{r}-\tilde{r}_{h}}{\tilde{r}_{h}}+\cdots .
$$

This can be inverted (as $\tilde{r} \rightarrow \tilde{r}_{h}, r \rightarrow-\infty$ ) to give

$$
\frac{\tilde{r}-\tilde{r}_{h}}{\tilde{r}_{h}}=e^{\sqrt{\Lambda} r}\left[1-\frac{7}{6} e^{\sqrt{\Lambda} r}+\cdots\right] .
$$

In the new coordinates the metric near $r=-\infty$ becomes

$$
\begin{aligned}
d s^{2}= & d r^{2}-\tilde{r}_{h}^{2} \Lambda e^{2 \sqrt{\Lambda} r}\left(1-\frac{14}{3} e^{\sqrt{\Lambda} r}+\cdots\right) d t^{2} \\
& +\tilde{r}_{h}^{2}\left(1+2 e^{\sqrt{\Lambda} r}+\cdots\right)\left(d x^{2}+d y^{2}+d z^{2}\right)
\end{aligned}
$$

and the gauge field becomes

$$
A=\tilde{r}_{h} \sqrt{\Lambda} \sqrt{2} e^{\sqrt{\Lambda} r}\left(1-\frac{8}{3} e^{\sqrt{\Lambda} r}+\cdots\right) d t .
$$

If we rescale the coordinates as $t \rightarrow \frac{t}{\tilde{r}_{h} \sqrt{\Lambda}}$ and $\{x, y, z\} \rightarrow \frac{1}{\tilde{r}_{h}}\{x, y, z\}$, then the solution up to first order in deviation near $r=-\infty$ can be written as, eq. (5.43), eq. (5.44).

Open Access. This article is distributed under the terms of the Creative Commons Attribution License which permits any use, distribution and reproduction in any medium, provided the original author(s) and source are credited.

\section{References}

[1] L.D. Landau and E.M. Lifshitz, The classical theory of fields, Pergamon Press, U.K. (1980).

[2] M.P. Ryan and L.C. Shepley, Homogeneous relativistic cosmologies, Princeton Series in Physics, Princeton University Press, Princeton U.S.A. (1975).

[3] M. Kataoka and O. Nakanishi, Helical spin density wave due to antisymmetric exchange interaction, J. Phys. Soc. Jpn. 50 (1981) 3888.

[4] M.L. Plumer and M.B. Walker, Wavevector and spin reorientation in MnSi, J. Phys. C 14 (1981) 4689.

[5] P. Fulde and R.A. Ferell, Superconductivity in a strong spin-exchange field, Phys. Rev. 135 (1964) A550.

[6] A.I. Larkin and Y.N. Ovchinnikov, Inhomogeneous state of superconductors, Sov. Phys. JETP 20 (1965) 762.

[7] D. Deryagin, D.Y. Grigoriev and V. Rubakov, Standing wave ground state in high density, zero temperature QCD at large- $N_{c}$, Int. J. Mod. Phys. A 7 (1992) 659 [InSPIRE].

[8] S.A. Brazovskii, Phase transition of an isotropic system to a nonuniform state, Sov. Phys. JETP 41 (1975) 85. 
[9] S.K. Domokos and J.A. Harvey, Baryon number-induced Chern-Simons couplings of vector and axial-vector mesons in holographic QCD, Phys. Rev. Lett. 99 (2007) 141602 [arXiv: 0704.1604] [INSPIRE].

[10] S. Nakamura, H. Ooguri and C.-S. Park, Gravity dual of spatially modulated phase, Phys. Rev. D 81 (2010) 044018 [arXiv:0911.0679] [inSPIRE].

[11] H. Ooguri and C.-S. Park, Holographic end-point of spatially modulated phase transition, Phys. Rev. D 82 (2010) 126001 [arXiv:1007.3737] [InSPIRE].

[12] H. Ooguri and C.-S. Park, Spatially modulated phase in holographic quark-gluon plasma, Phys. Rev. Lett. 106 (2011) 061601 [arXiv:1011.4144] [INSPIRE].

[13] A. Donos and J.P. Gauntlett, Holographic helical superconductors, JHEP 12 (2011) 091 [arXiv: 1109.3866] [INSPIRE].

[14] A. Donos, J.P. Gauntlett and C. Pantelidou, Spatially modulated instabilities of magnetic black branes, JHEP 01 (2012) 061 [arXiv:1109.0471] [INSPIRE].

[15] A. Donos and J.P. Gauntlett, Holographic striped phases, JHEP 08 (2011) 140 [arXiv:1106.2004] [INSPIRE].

[16] D. Marolf, Black holes and branes in supergravity, arXiv:1107.1022 [INSPIRE].

[17] H.K. Kunduri, J. Lucietti and H.S. Reall, Near-horizon symmetries of extremal black holes, Class. Quant. Grav. 24 (2007) 4169 [arXiv:0705.4214] [InSPIRE].

[18] S.S. Gubser, Breaking an Abelian gauge symmetry near a black hole horizon, Phys. Rev. D 78 (2008) 065034 [arXiv:0801.2977] [INSPIRE].

[19] G.T. Horowitz, Introduction to holographic superconductors, arXiv:1002.1722 [INSPIRE].

[20] R. Gregory and R. Laflamme, Black strings and p-branes are unstable, Phys. Rev. Lett. 70 (1993) 2837 [hep-th/9301052] [INSPIRE].

[21] R. Gregory and R. Laflamme, The instability of charged black strings and p-branes, Nucl. Phys. B 428 (1994) 399 [hep-th/9404071] [INSPIRE].

[22] L. Lehner and F. Pretorius, Final state of Gregory-Laflamme instability, arXiv:1106.5184 [INSPIRE].

[23] S. Kachru, X. Liu and M. Mulligan, Gravity duals of Lifshitz-like fixed points, Phys. Rev. D 78 (2008) 106005 [arXiv:0808.1725] [INSPIRE].

[24] M. Taylor, Non-relativistic holography, arXiv:0812.0530 [INSPIRE].

[25] S.A. Hartnoll, J. Polchinski, E. Silverstein and D. Tong, Towards strange metallic holography, JHEP 04 (2010) 120 [arXiv:0912.1061] [INSPIRE].

[26] K. Balasubramanian and K. Narayan, Lifshitz spacetimes from AdS null and cosmological solutions, JHEP 08 (2010) 014 [arXiv: 1005.3291] [INSPIRE].

[27] A. Donos and J.P. Gauntlett, Lifshitz solutions of $D=10$ and $D=11$ supergravity, JHEP 12 (2010) 002 [arXiv: 1008.2062] [INSPIRE].

[28] R. Gregory, S.L. Parameswaran, G. Tasinato and I. Zavala, Lifshitz solutions in supergravity and string theory, JHEP 12 (2010) 047 [arXiv: 1009.3445] [INSPIRE].

[29] A. Donos, J.P. Gauntlett, N. Kim and O. Varela, Wrapped M5-branes, consistent truncations and $A d S / C M T, J H E P 12$ (2010) 003 [arXiv:1009.3805] [INSPIRE]. 
[30] D. Cassani and A.F. Faedo, Constructing Lifshitz solutions from AdS, JHEP 05 (2011) 013 [arXiv: 1102.5344] [INSPIRE].

[31] N. Halmagyi, M. Petrini and A. Zaffaroni, Non-relativistic solutions of $N=2$ gauged supergravity, JHEP 08 (2011) 041 [arXiv:1102.5740] [INSPIRE].

[32] S. Ferrara, R. Kallosh and A. Strominger, $N=2$ extremal black holes, Phys. Rev. D 52 (1995) 5412 [hep-th/9508072] [INSPIRE].

[33] S. Bellucci, S. Ferrara, R. Kallosh and A. Marrani, Extremal black hole and flux vacua attractors, Lect. Notes Phys. 755 (2008) 115 [arXiv:0711.4547] [INSPIRE].

[34] S. Ferrara, G.W. Gibbons and R. Kallosh, Black holes and critical points in moduli space, Nucl. Phys. B 500 (1997) 75 [hep-th/9702103] [INSPIRE].

[35] G.W. Gibbons, R. Kallosh and B. Kol, Moduli, scalar charges and the first law of black hole thermodynamics, Phys. Rev. Lett. 77 (1996) 4992 [hep-th/9607108] [INSPIRE].

[36] A. Sen, Black hole entropy function and the attractor mechanism in higher derivative gravity, JHEP 09 (2005) 038 [hep-th/0506177] [INSPIRE].

[37] K. Goldstein, N. Iizuka, R.P. Jena and S.P. Trivedi, Non-supersymmetric attractors, Phys. Rev. D 72 (2005) 124021 [hep-th/0507096] [INSPIRE].

[38] R. Kallosh, N. Sivanandam and M. Soroush, The non-BPS black hole attractor equation, JHEP 03 (2006) 060 [hep-th/0602005] [INSPIRE].

[39] S. Kachru, R. Kallosh and M. Shmakova, Generalized attractor points in gauged supergravity, Phys. Rev. D 84 (2011) 046003 [arXiv:1104.2884] [INSPIRE].

[40] C.M. Brown and O. DeWolfe, The Godel-Schrödinger spacetime and stringy chronology protection, JHEP 01 (2012) 032 [arXiv:1110.3840] [INSPIRE].

[41] G.T. Horowitz and B. Way, Lifshitz singularities, Phys. Rev. D 85 (2012) 046008 [arXiv: 1111.1243] [INSPIRE].

[42] C. Charmousis, B. Gouteraux, B. Kim, E. Kiritsis and R. Meyer, Effective holographic theories for low-temperature condensed matter systems, JHEP 11 (2010) 151 [arXiv: 1005.4690] [INSPIRE].

[43] E. Perlmutter, Domain wall holography for finite temperature scaling solutions, JHEP 02 (2011) 013 [arXiv: 1006.2124] [INSPIRE].

[44] N. Iizuka, N. Kundu, P. Narayan and S.P. Trivedi, Holographic Fermi and non-Fermi liquids with transitions in dilaton gravity, JHEP 01 (2012) 094 [arXiv:1105.1162] [INSPIRE].

[45] B. Gouteraux and E. Kiritsis, Generalized holographic quantum criticality at finite density, JHEP 12 (2011) 036 [arXiv:1107.2116] [INSPIRE].

[46] N. Ogawa, T. Takayanagi and T. Ugajin, Holographic Fermi surfaces and entanglement entropy, JHEP 01 (2012) 125 [arXiv:1111.1023] [INSPIRE].

[47] L. Huijse, S. Sachdev and B. Swingle, Hidden Fermi surfaces in compressible states of gauge-gravity duality, Phys. Rev. B 85 (2012) 035121 [arXiv:1112.0573] [INSPIRE].

[48] X. Dong, S. Harrison, S. Kachru, G. Torroba and H. Wang, Aspects of holography for theories with hyperscaling violation, JHEP 06 (2012) 041 [arXiv:1201.1905] [INSPIRE]. 